\title{
MicroRNA-210 overexpression promotes psoriasis-like inflammation by inducing Th1 and Th17 cell differentiation
}

\author{
Ruifang Wu, ${ }^{1}$ Jinrong Zeng, ${ }^{1}$ Jin Yuan, ${ }^{1}$ Xinjie Deng, ${ }^{1}$ Yi Huang, ${ }^{1}$ Lina Chen, ${ }^{1}$ Peng Zhang, ${ }^{1}$ Huan Feng, ${ }^{1}$ Zixin Liu, ${ }^{1}$ Zijun Wang, \\ Xiaofei Gao, ${ }^{1}$ Haijing Wu, ${ }^{1}$ Honglin Wang, ${ }^{2}$ Yuwen Su, ${ }^{1}$ Ming Zhao, ${ }^{1}$ and Qianjin Lu ${ }^{1}$
}

'Department of Dermatology, Hunan Key Laboratory of Medical Epigenomics, The Second Xiangya Hospital of Central South University, Changsha, Hunan, China. ${ }^{2}$ Shanghai Institute of Immunology, Key Laboratory of Cell Differentiation and Apoptosis of Chinese Ministry of Education, Shanghai Jiao Tong University School of Medicine (SJTU-SM), Shanghai, China.

Immune imbalance of T lymphocyte subsets is a hallmark of psoriasis, but the molecular mechanisms underlying this aspect of psoriasis pathology are poorly understood. Here, we report that microRNA-210 (miR-210), a miR that is highly expressed in both psoriasis patients and mouse models, induces helper T (Th) 17 and Th1 cell differentiation but inhibits Th2 differentiation through repressing STAT6 and LYN expression, contributing to several aspects of the immune imbalance in psoriasis. Both miR-210 ablation in mice and inhibition of miR-210 by intradermal injection of antagomir-210 blocked the immune imbalance and the development of psoriasis-like inflammation in an imiquimod-induced or IL-23-induced psoriasis-like mouse model. We further showed that TCF- $\beta$ and IL-23 enhance miR-210 expression by inducing HIF-1 $\alpha$, which recruits P300 and promotes histone $\mathrm{H} 3$ acetylation in the miR-210 promoter region. Our results reveal a crucial role for miR-210 in the immune imbalance of T lymphocyte subsets in psoriasis and suggest a potential therapeutic avenue.

\section{Introduction}

Psoriasis is a $\mathrm{T}$ cell-mediated inflammatory cutaneous disease (1). Immune imbalance of $\mathrm{T}$ cells is believed to play a key role in psoriasis pathogenesis (2). When genetically susceptible individuals are exposed to various environmental factors, such as microorganism infection and physical trauma, the inflammatory cascade in psoriatic plaques is triggered through plasmacytoid dendritic cells (pDCs) (3). The activated DCs then produce IL-23, IL-12, IL-6, and TNF- $\alpha$, which activate and polarize autoaggressive helper T (Th) cells toward Th17, Th1, and Th22 cell subsets defined by the production of IFN- $\gamma$, IL-17A, IL-17F, and IL-22, respectively (4-6). In turn, these proinflammatory cytokines act on keratinocytes and endothelial cells, leading to the activation, proliferation, and production of chemokines, adhesion molecules, and antimicrobial peptides; these changes result in further recruitment of leukocytes and amplify the immune response in psoriatic plaques (7-9). Based on the underlying pathogenesis, several therapies targeting these cells and cytokines, such as etanercept (10), ustekinumab (11), and secukinumab (12), have been developed. Although these monoclonal antibodies (Abs) have resulted in strong improvements in patients with psoriasis, there are remaining issues: the single effect, exorbitant price, and uncertainty of long-term antagonism of just one cytokine. Thus, further elucidation of $\mathrm{T}$ lymphocyte subset dysfunction and the molecular mechanisms in psoriasis, as well as identification of

Authorship note: RW and JZ contributed equally to this work. Conflict of interest: The authors have declared that no conflict of interest exists. Submitted: September 12, 2017; Accepted: March 20, 2018. Reference information: J Clin Invest. 2018;128(6):2551-2568. https://doi.org/10.1172/JCI97426. efficient new targets with multiple effects are the subject of considerable debate and ongoing investigation.

MicroRNAs (miRs) are small endogenous noncoding RNA molecules composed of approximately 22 nucleotides that negatively regulate gene expression at the posttranscriptional level by promoting target mRNA degradation and/or translational repression (13). Hundreds of miRs are expressed in keratinocytes and immune cells and play essential roles in regulating their development and function (14-17). Expression profiling studies have revealed that many miRs, such as miR-203 (18), miR-31 (16), and miR-138 (19), are dysregulated in keratinocytes and lymphocytes from patients with psoriasis. In our previous studies, we found that miR-210, a signature miR of hypoxia, was significantly upregulated in the peripheral blood mononuclear cells (PBMCs) and $\mathrm{CD} 4^{+} \mathrm{T}$ cells of psoriasis patients compared with healthy controls (20). Overexpression of miR-210 contributed to aberrant inflammatory cytokine expression in $\mathrm{CD}^{+} \mathrm{T}$ cells (20). Nevertheless, the role of miR-210 overexpression, the regulatory mechanisms, and in vivo physiological significance in the development of psoriasis remain poorly understood.

In this study, we report that increased expression of miR-210 enhanced Th1 and Th17 cells in peripheral blood and skin lesions of psoriasis patients and psoriasis-like mouse models by inhibiting its targets STAT6 and LYN, which aggravated skin lesions of psoriasis. Either genetic knockout of miR-210 or silencing of miR-210 by intradermal injection of the miR-210 inhibitor antagomir-210 prevented the development of psoriasis-like inflammation in the imiquimod-induced (IMQ-induced) or IL-23-induced psoriasislike mouse model, indicating a potential therapeutic target for psoriasis. Furthermore, we also revealed that the increased expression of HIF-1 $\alpha$ induced by the inflammatory cytokines, such 
A

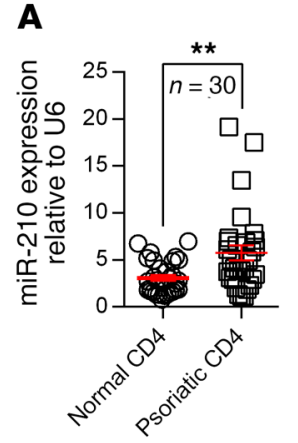

B

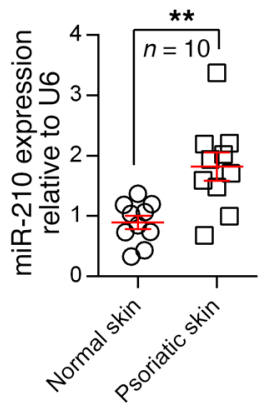

C

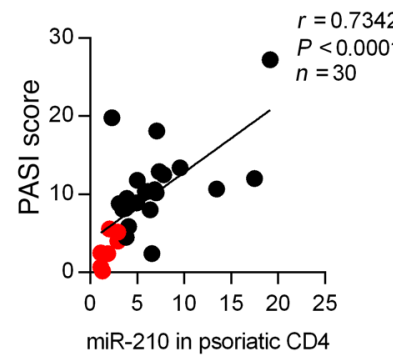

D

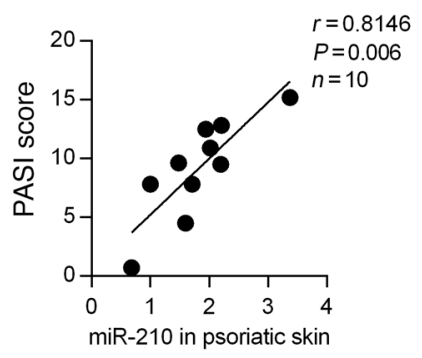

E
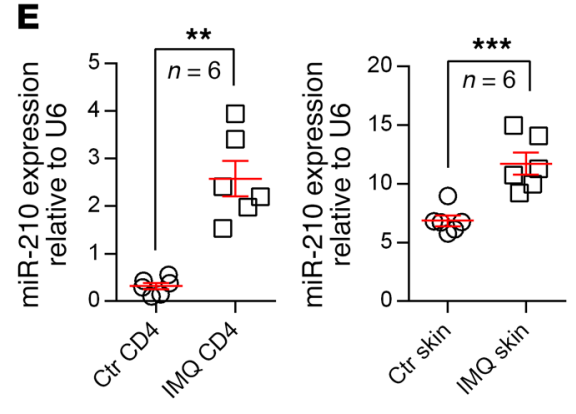

$\mathbf{F}$

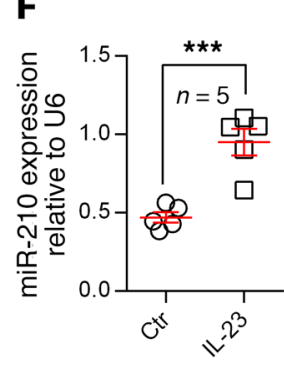

H

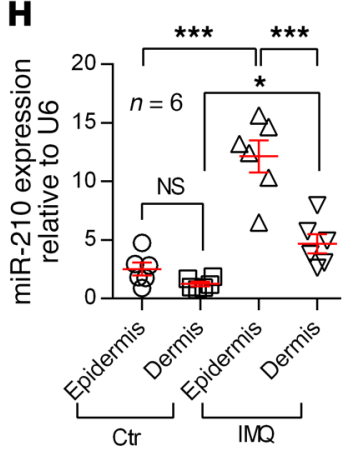

G
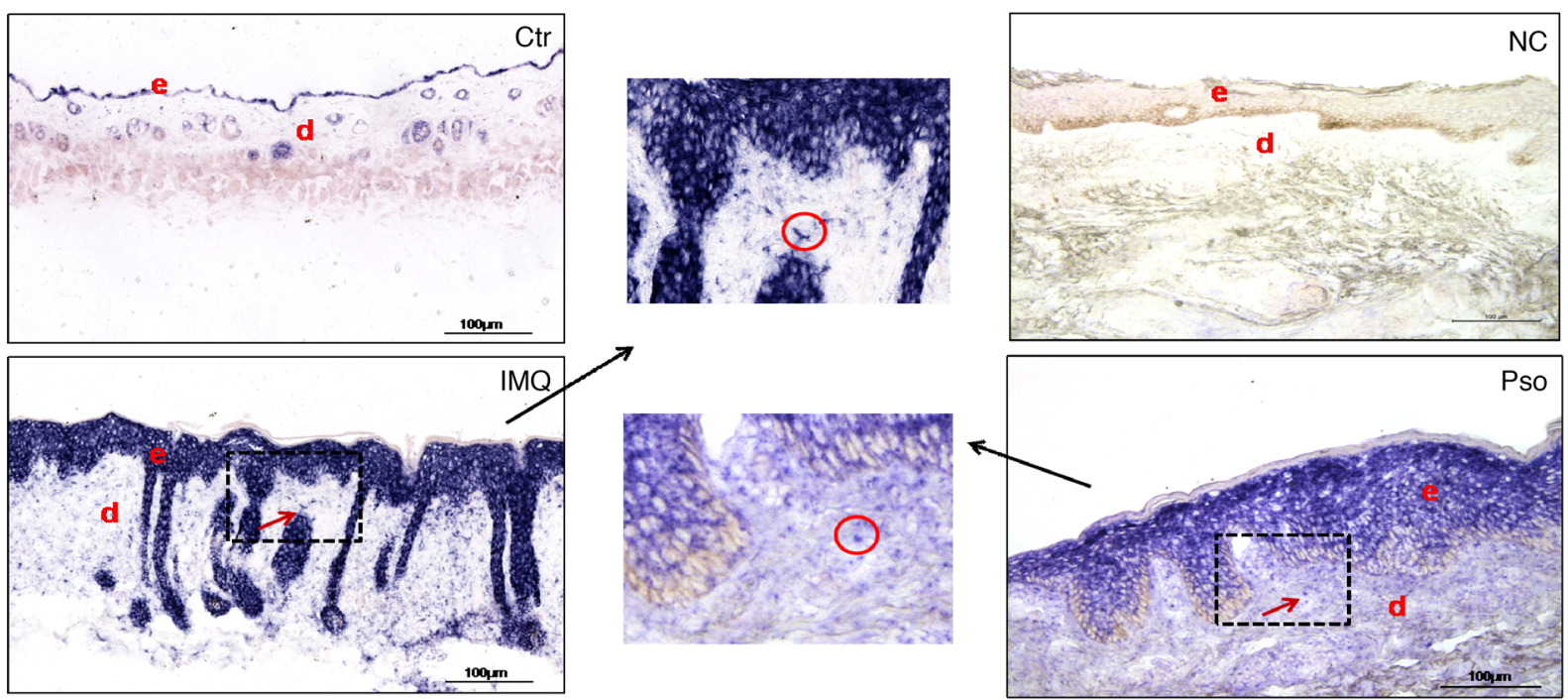

I

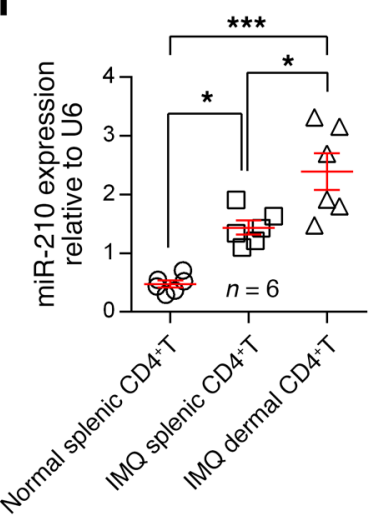

J
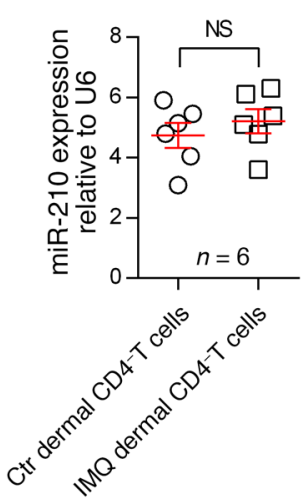
Figure 1. miR-210 expression is elevated in psoriatic $\mathrm{CD4} 4^{+} \mathrm{T}$ cells and skin lesions. ( $\mathbf{A}$ and $\mathbf{B}$ ) Expression of miR-210 in CD4 ${ }^{+}$T cells $(\mathbf{A}, n=30)$ and skin samples (B, $n=10$ ) derived from psoriasis patients and healthy controls. (C and $\mathbf{D})$ Correlation of human miR-210 expression in psoriatic $C D 4^{+} \mathrm{T}$ cells $(\mathbf{C}, n=30)$ or skin $(\mathbf{D}, n=10)$ with PASI scores. (E) Expression of miR-210 in splenic CD4 ${ }^{+}$T cells (left panel) and skin samples (right panel) from untreated mice $(n=6)$ and IMQ-induced mice $(n=6)$. (F) Expression of miR-210 in skin lesions from normal ears $(n=5)$ and IL-23-treated ears $(n=5)$ of mice. (G) In situ hybridization was performed on mouse skin treated with vehicle (Ctr, $n=5)$ or IMQ (IMQ, $n=5)$ as well as human skin from healthy controls (NC, $n=6$ ) and psoriasis patients (Pso, $n=5$ ) using miR-210-specific LNA probes. Dark blue color indicates miR-210 expression. e, epidermis; d, dermis. Scale bars: $100 \mu \mathrm{m}$ (lower magnification) and $50 \mu \mathrm{m}$ (higher magnification). Red arrows indicate dermis-infiltrating inflammatory cells. (H) Expression of miR-210 in the epidermis and dermis of skin samples from untreated controls $(n=6)$ or IMQ-treated mice $(n=6)$. (I) miR-210 levels in CD4+ $T$ cells derived from untreated mouse spleen $(n=6)$, IMQ-treated mouse spleen $(n=6)$, and IMQ-treated mouse lesional dermis $(n=6)$. (J) Expression of miR-210 in dermal CD4- T cells of skin samples from untreated controls $(n=6)$ or IMQ-treated mice $(n=6)$. Data are pooled from 2 independent experiments (A-D) or are representative of 3 independent experiments (E-J) in BALB/C mice (E and $\mathbf{G}-\mathrm{J}$ ) and in C57BL/6J mice (F). Data represent the mean \pm SEM. ${ }^{*} P<0.05$, ${ }^{*} P<0.01$, ${ }^{* *} P<0.001$. Twotailed Mann-Whitney $U$ test (A and B), 2-tailed unpaired Student's $t$ test $(\mathbf{E}, \mathbf{F}$, and $\mathbf{J})$, Spearman's $r$ test (C and $\mathbf{D})$, or 1-way ANOVA with Bonferroni's post hoc test ( $\mathbf{H}$ and $\mathbf{I})$ was used.

as TGF- $\beta$ and IL-23, enhanced miR-210 expression in CD $4^{+} \mathrm{T}$ cells and skin lesions of psoriasis through epigenetic mechanisms.

\section{Results}

miR-210 expression is elevated in psoriatic $C D 4^{+} T$ cells and skin lesions. We measured miR-210 expression in peripheral blood $\mathrm{CD}^{+} \mathrm{T}$ cells and skin lesions from psoriasis patients and found that it is upregulated in both $\mathrm{CD} 4^{+} \mathrm{T}$ cells and skin lesions from psoriasis patients compared with healthy controls (Figure 1, A and B). Notably, we observed no significant increase in miR-210 expression in $\mathrm{CD}^{+} \mathrm{T}$ cells of a fraction of psoriasis patients with low clinical scores, which is consistent with the positive correlation between the miR-210 expression levels in $\mathrm{CD}^{+} \mathrm{T}$ cells or lesional skins and the psoriasis area and severity index (PASI) scores of psoriasis patients (Figure 1, C and D). In addition, we established IMQ- and IL-23-induced psoriasis-like mouse models, which closely resemble the human disease phenotype according to previously published studies $(16,21,22)$. As expected, the IMQ- or IL-23-treated mice developed typical psoriasis-like lesions with evident clinical and pathological changes (Supplemental Figures 1-3; supplemental material available online with this article; https://doi.org/10.1172/JCI97426DS1). Consistent with the results of human samples, mice exposed to IMQ or IL-23 expressed significantly higher levels of miR-210 in splenic CD $4^{+}$ $\mathrm{T}$ cells and lesional skins compared with matrix-exposed mice (Figure 1, E and F). Similar to the psoriasis-like mouse model, an experimental autoimmune encephalomyelitis (EAE) mouse model (a Th1 cell- and Th17 cell-mediated ideal mouse model for human multiple sclerosis) also exhibited significantly increased miR-210 expression (Supplemental Figure 4).

To determine whether miR-210 was increased in infiltrating $\mathrm{CD}^{+} \mathrm{T}$ cells from the psoriatic skin lesions, we performed in situ hybridization on skin cryosections from psoriasis patients and the IMQ-treated mice using a miR-210-specific locked nucleic acid-modified (LNA) probe. The results showed that miR-210 expression was elevated in the dermis, as well as in the epidermis (Figure 1G), which was consistent with the reverse transcription quantitative PCR (RT-qPCR) analysis of the epidermis and dermis of skin lesions (Figure $1 \mathrm{H}$ ). Furthermore, we sorted CD $4^{+}$ $\mathrm{T}$ cells from dermal single-cell suspensions of IMQ-induced psoriasis-like skin lesions and confirmed that miR-210 was specifically elevated in dermal $\mathrm{CD}^{+} \mathrm{T}$ cells and not in dermal CD4- ${ }^{-}$cells (Figure 1, I and J, and Supplemental Figure 5). These data demonstrated that the elevated miR-210 expression may be functionally involved in the $\mathrm{CD}^{+} \mathrm{T}$ cell-mediated immune dysfunction in peripheral and skin lesions of psoriasis.

miR-210 skews the CD4 $4^{+}$Th cell-mediated immune balance in psoriasis. The imbalance of Th cells, such as Th1/Th2 and Th17/Treg, was shown to play an important role in psoriasis pathogenesis (23). To determine whether the upregulation of miR-210 contributes to the immune imbalance of psoriasis, we first investigated miR-210 expression in different $\mathrm{T}$ cell subsets. We activated human naive $\mathrm{CD}^{+} \mathrm{T}$ cells under different polarizing conditions in vitro (Supplemental Figure 6) and found significantly increased miR-210 expression in Th17 cells and slightly increased miR-210 expression in Th1 cells compared with Th0 cells (Figure 2A), suggesting that miR-210 might have priority to regulate Th17 and Th1 cell differentiation. Agomirs or antagomirs are modified oligonucleotides with improved stability and delivery that induce miR overexpression or silencing, respectively (24-26). To directly assess whether miR-210 regulates Th cell differentiation, we transfected naive human $\mathrm{CD} 4^{+} \mathrm{T}$ cells with agomir-210 or negative control agomir (agomir-NC) and then cultured the cells under Th1-, Th2-, Th17-, and inducible Treg-polarizing (iTreg-polarizing) conditions in vitro. The results showed that the increased miR-210 expression promoted Th17 and Th1 differentiation but inhibited Th2 differentiation (Figure 2, B and C). By contrast, in vitro differentiation of Th17 and Th1 was significantly suppressed and Th2 differentiation was increased by transfection with antagomir-210, which downregulated miR-210 expression in T cells (Figure 2, B and D). miR-210 expression changes had no effect on the differentiation of iTreg cells (Figure 2, C and D). We observed a similar effect of miR-210 on $\mathrm{T}$ cell differentiation of naive mouse $\mathrm{CD} 4^{+}$ $\mathrm{T}$ cells following transfection of the cells with agomir-210 and antagomir-210 (Supplemental Figure 7).

Our studies also found that psoriatic $\mathrm{CD} 4^{+} \mathrm{T}$ cells and skin showed preferential expression of Th1- and Th17-related cytokines (Supplemental Figure 8). To investigate the effect of miR210 on cytokine secretion of Th cells, we transfected human psoriatic $\mathrm{CD}^{+} \mathrm{T}$ cells and normal human $\mathrm{CD} 4^{+} \mathrm{T}$ cells with antagomir-210 or agomir-210. We found that the production of IL-17A, IL-17F, and IFN- $\gamma$ in the supernatants was increased in normal $\mathrm{CD}^{+} \mathrm{T}$ cells when transfected with agomir-210 but significantly decreased in psoriatic $\mathrm{CD}^{+} \mathrm{T}$ cells transfected with antagomir-210 (Figure 2, E and F). By contrast, the IL-4 protein level in the supernatant of $\mathrm{CD} 4^{+} \mathrm{T}$ cells was decreased by transfection with agomir-210 and elevated by transfection with antagomir-210 (Figure 2, E and F). Similar changes were observed in the mRNA expression of cytokine genes (Figure 2, $\mathrm{G}$ and $\mathrm{H})$. Together, these data indicated that miR-210 promotes 
A

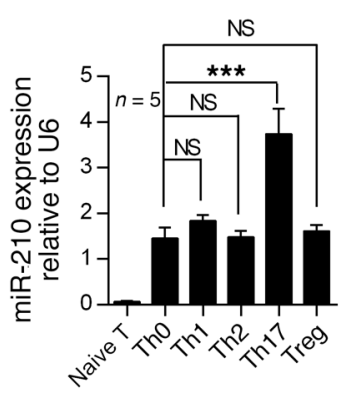

B

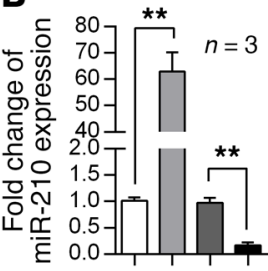

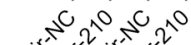

$50,590 \times 1090$
C
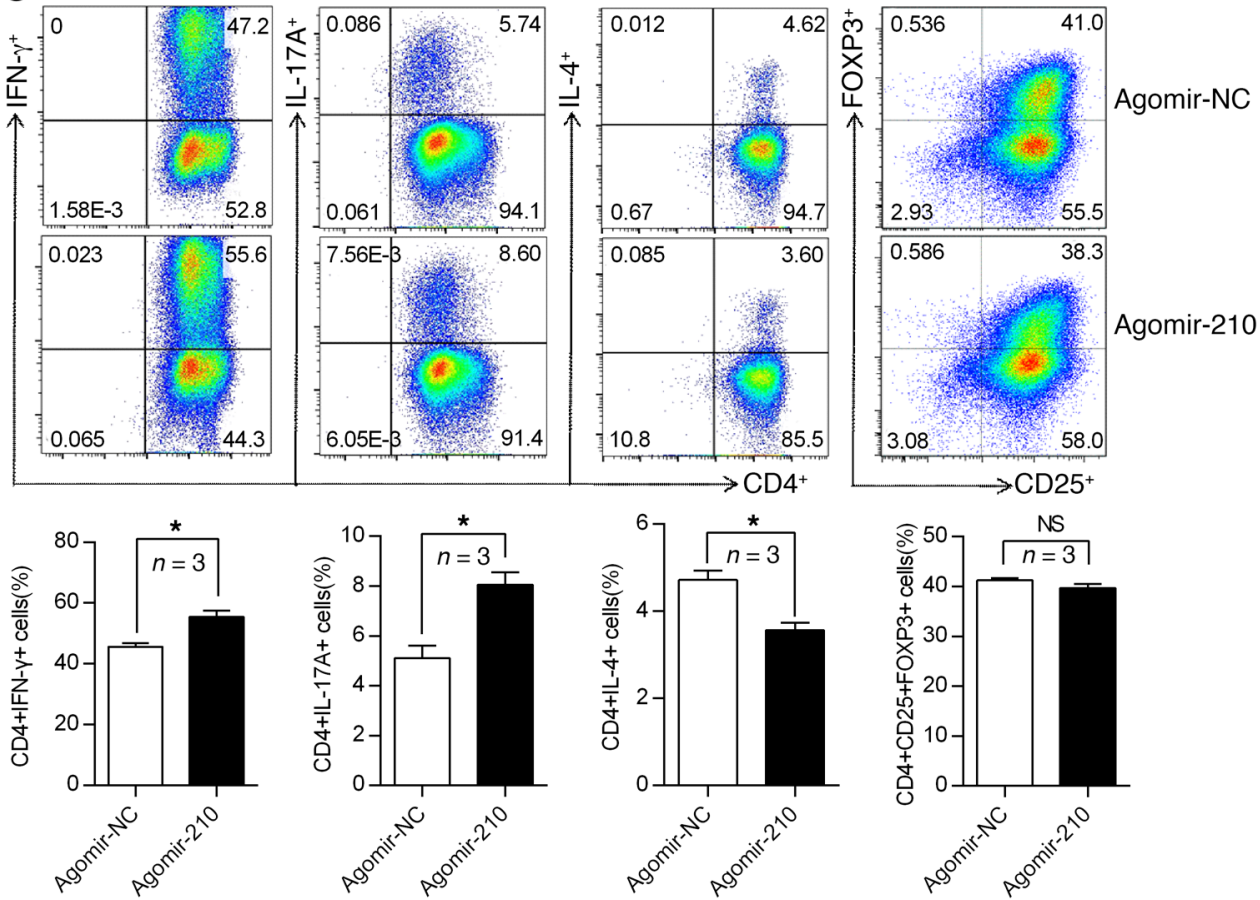
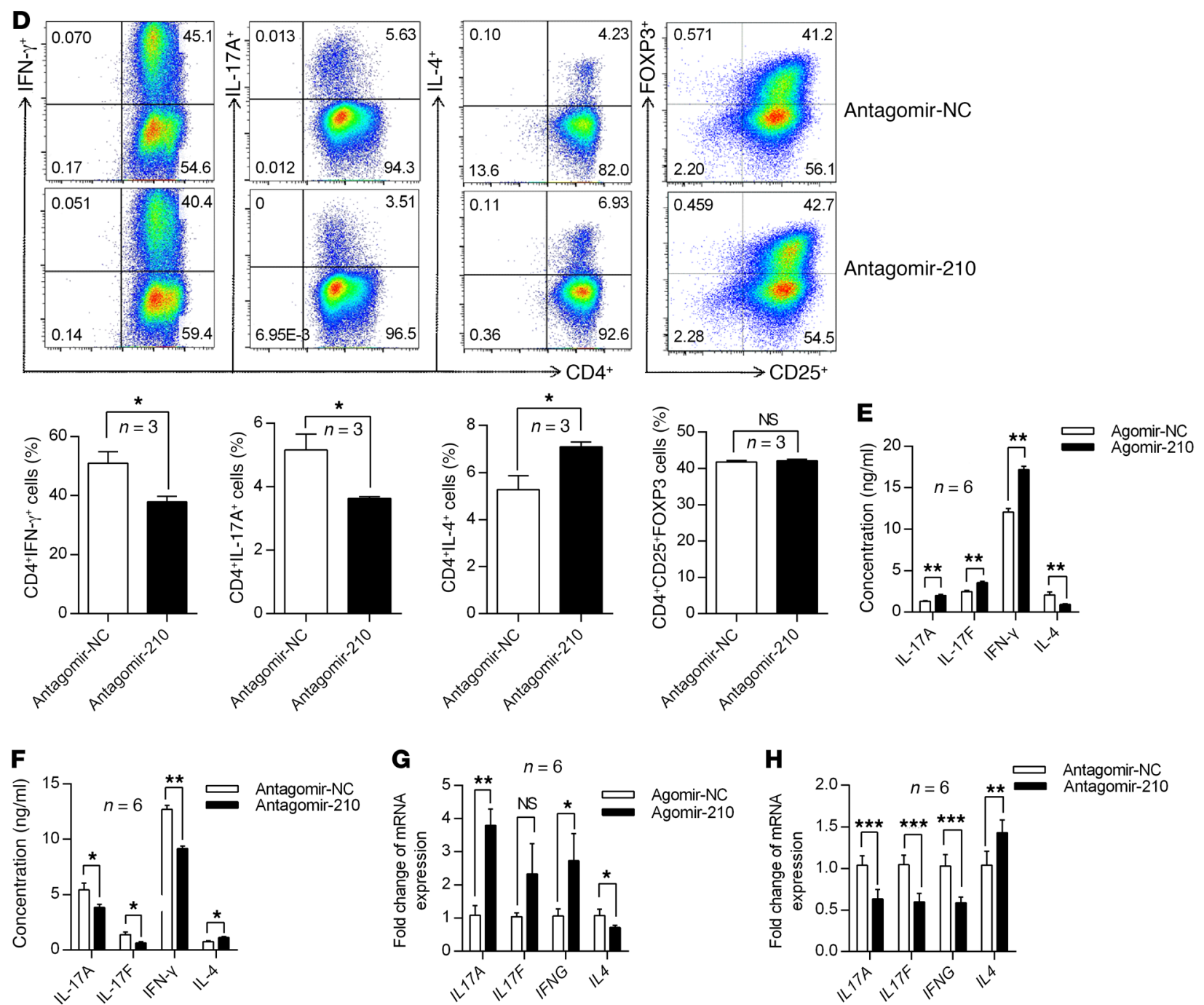
Figure 2. miR-210 contributes to the altered balance between pathogenic Th1/Th17 cells and Th2 cells in psoriasis. (A) miR-210 expression in naive CD4 ${ }^{+}$T, Th0, Th1, Th2, Th17, and iTreg cells $(n=5)$. (B) miR-210 expression in $\mathrm{CD}^{+}$T cells transfected with agomir-210 $(n=3)$ or antagomir-210 $(n=3)$. (C and D) Human naive $\mathrm{CD} 4^{+} \mathrm{T}$ cells were transfected with agomir-210 (C, $n=3)$, antagomir-210 ( $\mathbf{D}, n=3)$, or their corresponding controls and then were differentiated into Th1, Th2, Th17, and iTreg cells. The percentage of Th1, Th2, Th17, and iTreg cells was detected by flow cytometry. Statistical analysis data are shown in the lower panel. ( $E$ and $\mathbf{F}$ ) The protein levels of IL-17A, IL-17F, IFN- $\gamma$, and IL-4 in cultured supernatants from normal human $\mathrm{CD4}^{+} \mathrm{T}$ cells transfected with agomir-210 or agomir-NC $(\mathbf{E}, n=6)$ and psoriatic $C D 4^{+} T$ cells transfected with antagomir-210 or antagomir-NC $(F, n=6)$. ( $\mathbf{G}$ and $\mathbf{H}$ ) The mRNA levels of IL17A, IL17F, IFNG, and IL4 in normal human $\mathrm{CD}^{+} \mathrm{T}$ cells transfected with agomir-210 or agomir-NC $(\mathbf{G}, n=6)$ and psoriatic CD4 ${ }^{+} T$ cells transfected with antagomir-210 or antagomir-NC $(\mathbf{H}$, $n=6$ ). All experiments were performed in triplicate. Data represent the mean \pm SEM. ${ }^{*} P<0.05,{ }^{* *} P<0.01,{ }^{* * *} P<0.001$. NS, not significant. Oneway ANOVA with Dunnett's post hoc test (A) or 2-tailed unpaired Student's $t$ test (B-H) was used.

the differentiation and functions of Th17 and Th1 cells but has the opposite effect on Th2 subsets.

miR-210 accelerates the development of psoriasis. To investigate the functional relevance of miR-210 upregulation in the development of psoriasis, we injected agomir-210 or agomir-NC intradermally once per day for 4 consecutive days in the IMQ-induced psoriasis-like mouse model, and the mice were sacrificed at 4,7,10, and 14 days (Figure $3 \mathrm{~A}$ ). Compared with the agomir-NC group, the agomir-210 group showed accelerated psoriasis-like pathological progression and increased disease severity (Figure 3B). Over the course of the experiment, we found that the miR-210 levels were much higher on day 4 and day 7 in the agomir-210 group than the agomir-NC group, along with more severe clinical and pathological changes (Figure $3 \mathrm{C}$ ). On day 7 , the peak of psoriasis-like changes, we observed that the splenomegaly of mice in the agomir-210 group was more obvious than that in the agomir-NC group (Figure 3D). In addition, elevation of miR-210 by agomir- 210 significantly increased the disease severity, acanthosis, and dermal inflammatory cell infiltration (Figure 3, E-G). To confirm the increased inflammatory cell infiltration in dermis from the agomir-210 group, we obtained dermal single-cell suspensions and analyzed the percentages of Th1 and Th17 cells. We found that the percentages of both Th1 and Th17 cells that infiltrated the dermis from agomir-210-treated mice were increased significantly compared with the agomir-NC group (Supplemental Figure 9). Moreover, enhanced Il17a, Ill7f, and Ifng mRNA expression accompanied by decreased $\mathrm{Il} 4 \mathrm{mRNA}$ expression was observed in splenic $\mathrm{CD} 4^{+} \mathrm{T}$ cells of the agomir-210 group (Figure $3 \mathrm{H}$ ). These results demonstrated that miR-210 upregulation promotes and aggravates the development of psoriasis.

miR-210 deletion blocks psoriasis development. To further clarify the role of miR-210 in psoriasis development, we generated miR210-knockout (miR-210-KO) mice. The miR-210-KO mice were born normally and did not show any abnormality in body size and weight or in lymphocyte populations compared with age-matched wild-type (WT) mice. RT-qPCR analysis confirmed the miR-210 ablation in both splenic $\mathrm{CD} 4^{+} \mathrm{T}$ cells and skin of KO mice (Figure $4 \mathrm{~A})$. We then applied IMQ to both KO mice and WT mice and found that miR-210-KO mice were unable to develop typical psoriasis-like dermatitis with regard to both clinical manifestations and pathological changes (Figure 4B). In addition, the splenomegaly of KO mice was not obvious in IMQ-treated mice (Figure 4C). Strikingly, according to histological analysis of skin treated with IMQ, the acanthosis and inflammatory cell infiltration in the dermis were significantly improved in KO mice compared with that in WT mice (Figure 4D). Furthermore, we observed that the immune imbalance in splenic $\mathrm{CD} 4^{+} \mathrm{T}$ cells was less pronounced in KO mice compared with WT mice following treatment with IMQ (Figure 4E). We then stimulated naive $\mathrm{CD}^{+} \mathrm{T}$ cells of $\mathrm{WT}$ and KO mice under different polarizing conditions in vitro and found that naive $\mathrm{CD}^{+} \mathrm{T}$ cell differentiation toward Th1 and Th17 cells in KO mice was dramatically limited compared with that in WT mice. By contrast, the proportion of Th2 cells was significantly increased (Supplemental Figure 10). We also established an IL-23-induced psoriasis-like mouse model in miR-210-KO mice and WT mice. As expected, the WT mice developed more serious psoriasis-like dermatitis compared with KO mice based on both clinical manifestations and pathological changes (Supplemental Figure 11). These data indicated that miR-210 deletion prevents psoriasis by regulating $\mathrm{T}$ cell differentiation.

As we know, both activated immune cells and keratinocytes together contribute to the psoriatic skin lesion. To investigate the critical role of miR-210 deletion in immune cells or keratinocytes in the development of psoriatic lesion, we first generated bone marrow (BM) chimeric mice using miR-210-KO mice and WT mice. Lethally irradiated miR-210-KO or WT mice were adoptively transferred with BM from WT mice. The recipients of both genotypes (WT BM-KO and WT BM-WT) developed comparable levels of severity of psoriasis-like lesion after treatment with IMQ (Supplemental Figure 12, A-D). However, following the application of IMQ, the WT recipient mice, which were reconstituted with the BM from miR-210-KO mice (KO BM-WT), showed significantly alleviated skin lesions compared with WT BM-KO or WT BM-WT mice (Supplemental Figure 12, A-D). Furthermore, to demonstrate that the deletion of miR-210 in $\mathrm{CD}^{+} \mathrm{T}$ cells plays an important role in the pathogenesis of psoriasis, we transferred naive $\mathrm{CD} 4^{+} \mathrm{T}$ cells from miR-210-KO or WT mice to Rag2/- mice. As expected, Rag2 ${ }^{--}$ mice could not develop typical psoriasis-like dermatitis induced by IMQ (Supplemental Figure 12, E-H). More importantly, Rag2- mice transferred with naive $\mathrm{CD} 4^{+} \mathrm{T}$ cells with miR-210 deletion displayed notably improved IMQ-induced skin lesions compared with mice transferred with naive $\mathrm{CD} 4^{+} \mathrm{T}$ cells from WT mice (Supplemental Figure 12, E-H). Together, these findings indicated that the aberrant activation and differentiation of $\mathrm{CD} 4^{+} \mathrm{T}$ cells induced by miR-210 overexpression play a much more important role in the pathogenesis of psoriasis and miR-210 deletion in T cells is the major cause of notable improvement of skin lesion induced by IMQ.

Intradermal administration of antagomir-210 ameliorates the pathological phenotype of IMQ-induced psoriasis-like mice. Our findings indicated the important role of miR-210 in psoriasis pathogenesis. Therefore, we sought to evaluate the efficacy and potential application of a miR-210-based therapy for psoriasis. We injected antagomir-210 or antagomir-NC intradermally 4 times from the beginning to the third day to test the inhibitory effect on disease development in the IMQ-induced psoriasis-like mouse model (Figure 5A). As expected, miR-210 expression in both splenic CD ${ }^{+}$ 
A

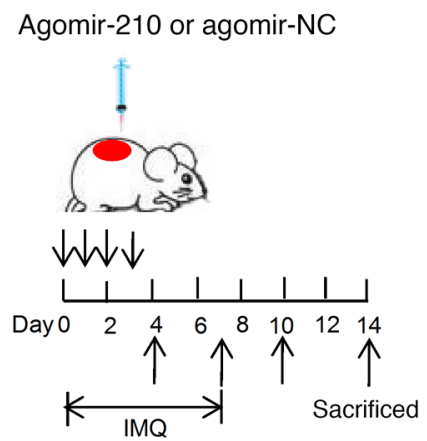

\section{C}

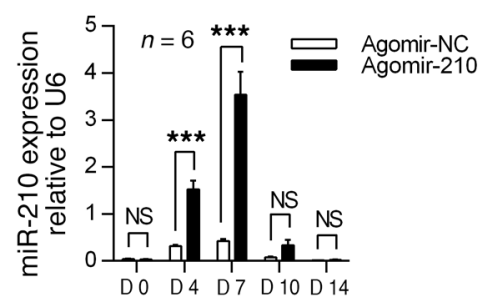

D

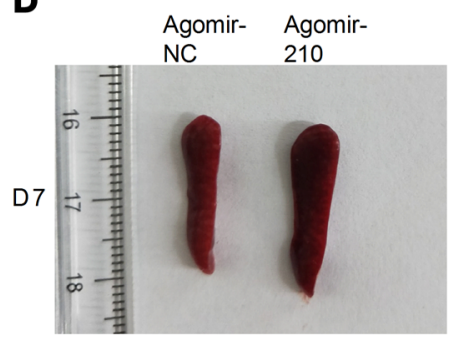

B

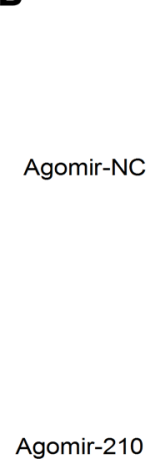

Agomir-NC

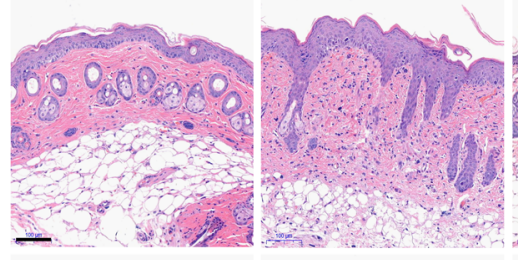

Agomir-210

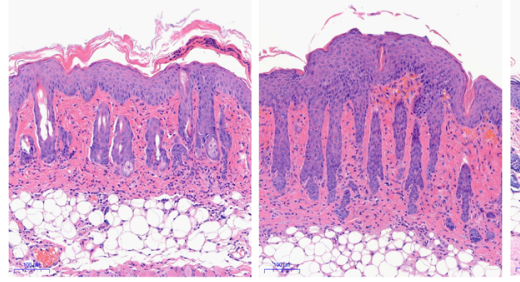

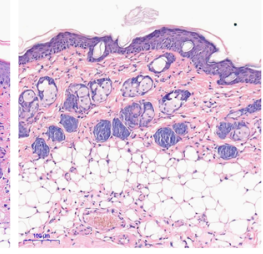

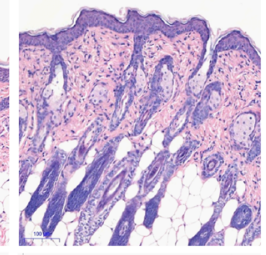

D 10
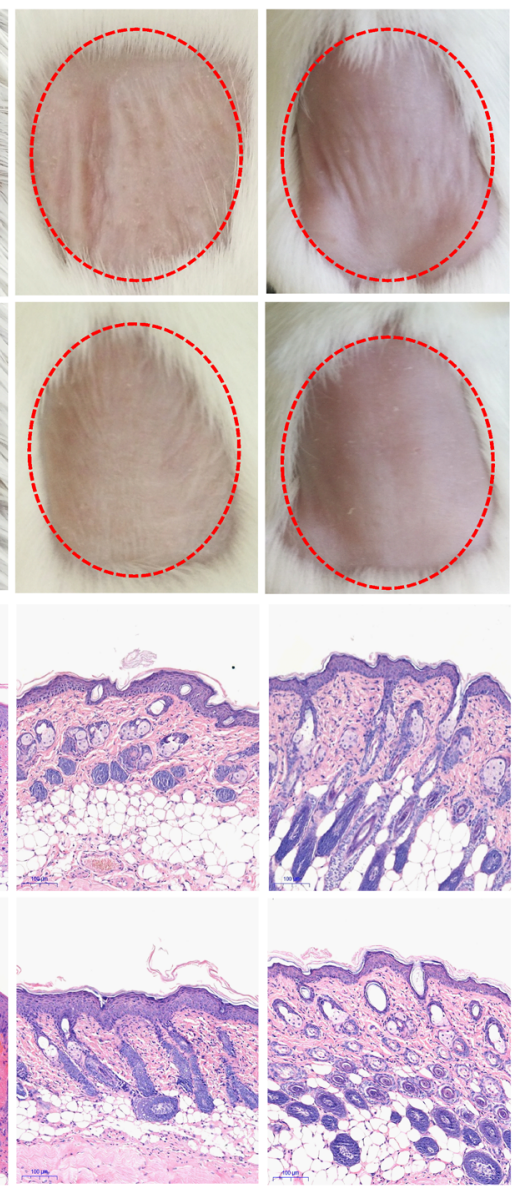

E

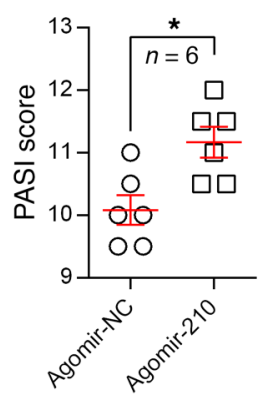

F

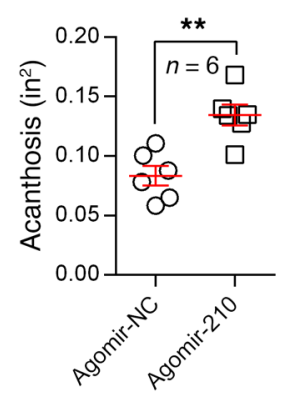

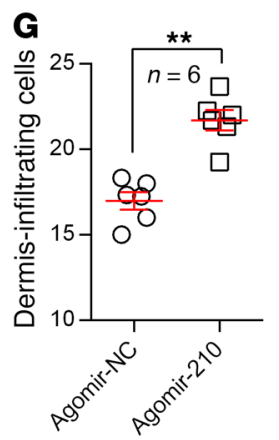

H

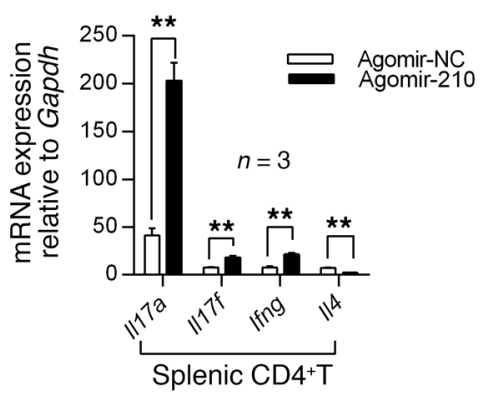

Figure 3. miR-210 accelerates the development of psoriasis. (A) Schematic diagram of intradermal administration of agomir-NC ( $5 \mathrm{nmol}$ ) or agomir-210 ( $5 \mathrm{nmol}$ ) on days $0,1,2$, and 3 during the application of IMQ in mice (BALB/c). Three mice in each group were sacrificed on days $4,7,10$, and 14 to conduct experiments. (B) Phenotypic presentation and H\&E staining of lesional skin from mice injected with agomir-NC and agomir-210. Scale bars: $100 \mu \mathrm{m}$. (C) The miR-210 expression in splenic CD4 ${ }^{+}$T cells from agomir-NC-treated $(n=6)$ or agomir-210-treated mice $(n=6)$. (D) The size of spleens of mice in A. (E) PASI scores of mice in $\mathbf{A}(n=6)$. ( $\mathbf{F}$ and $\mathbf{G}$ ) Acanthosis and dermal cellular infiltrates were quantified for mice treated with agomir-NC $(n=6)$ or agomir-210 $(n=6)$. For all measurements in $\mathbf{G}$, the median number of specifically stained dermal nucleated cells was counted in 3 high-power fields per section. (H) The mRNA levels of $/ 117 a$, II17f, Ifng, and II4 in splenic CD4+ T cells from agomir-NC-treated $(n=3)$ or agomir-210-treated mice $(n=3)$. Data $(\mathbf{D}-\mathbf{H})$ were obtained from agomir-NC- or agomir-210-treated mice on the seventh day. Data $(\mathbf{B}-\mathbf{H})$ are representative of at least 3 independent experiments with 3 to 6 samples per group in each. Data represent the mean $\pm \mathrm{SEM}$. ${ }^{*} P<0.05,{ }^{* *} P<0.01,{ }^{* * *} P<0.001$. NS, not significant. Two-tailed unpaired Student's $t$ test (C and $\mathbf{E}-\mathbf{H}$ ) was used.

$\mathrm{T}$ cells and skin lesions was dramatically reduced (Figure 5B), and there was a significant improvement in both clinical and pathological characteristics on day 7 following antagomir-210 treatment (Figure 5C), along with alleviated disease severity (Figure 5D) and splenomegaly (Figure 5E). In addition, the acanthosis and dermal inflammatory cell infiltration were significantly decreased after antagomir-210 administration (Figure 5F).

We also obtained splenic $\mathrm{CD}^{+} \mathrm{T}$ cells to assess the in vivo effect of antagomir-210 on the immune imbalance of Th cell subsets in psoriasis-like mouse models. Notably, silencing miR-210 
A
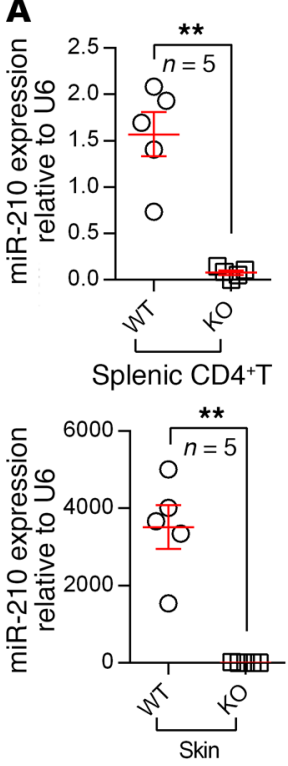

B
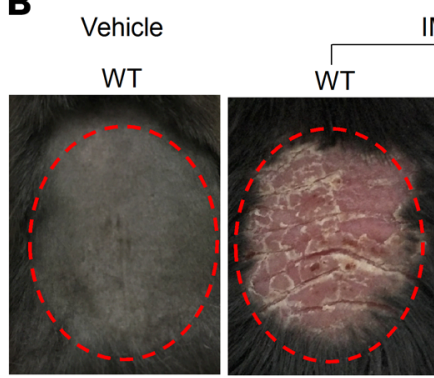

$\mathrm{MQ}$
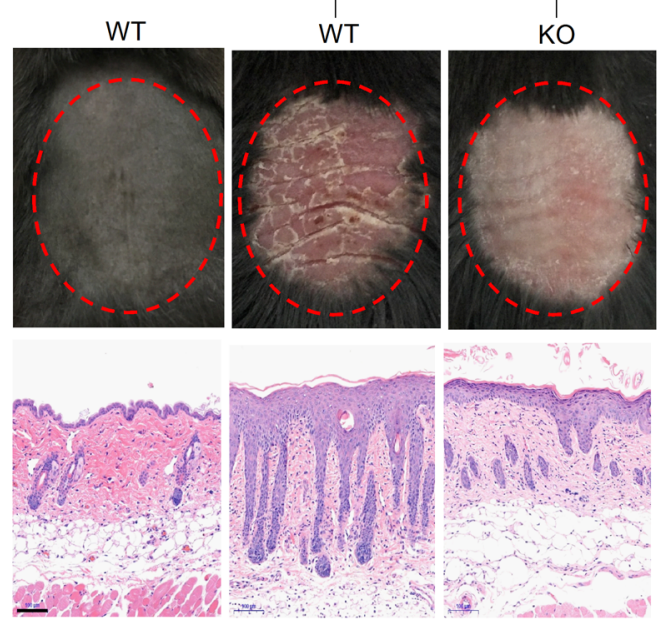

C

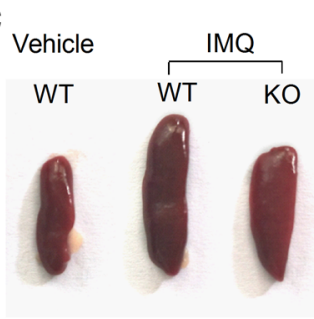

D

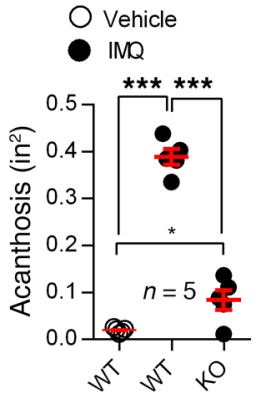

Vehicle
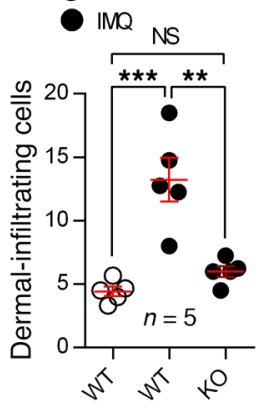

E
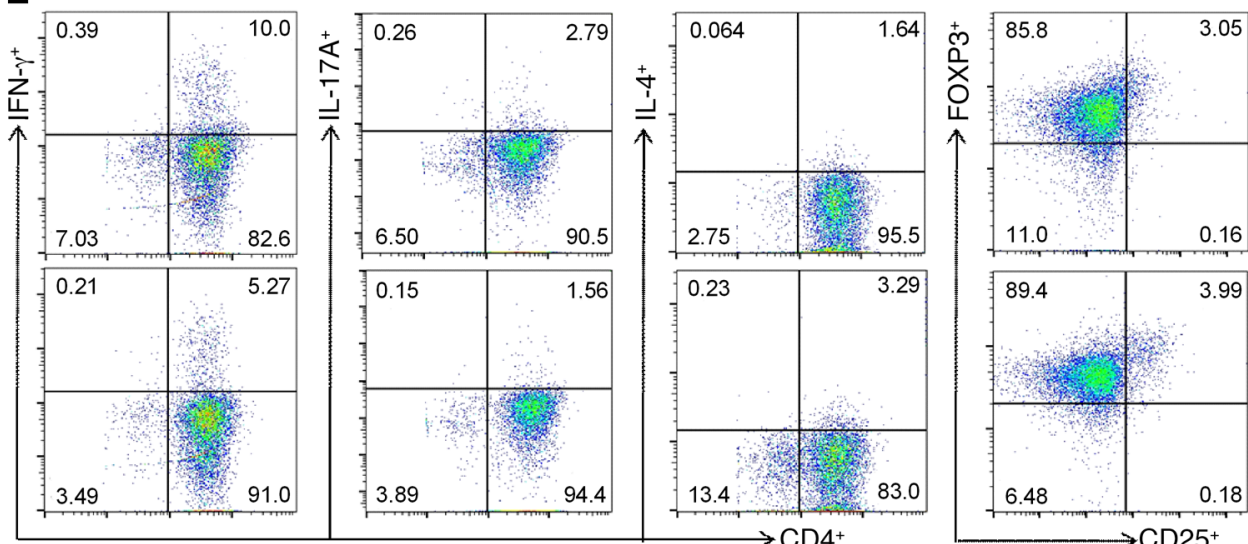

WT (IMQ)
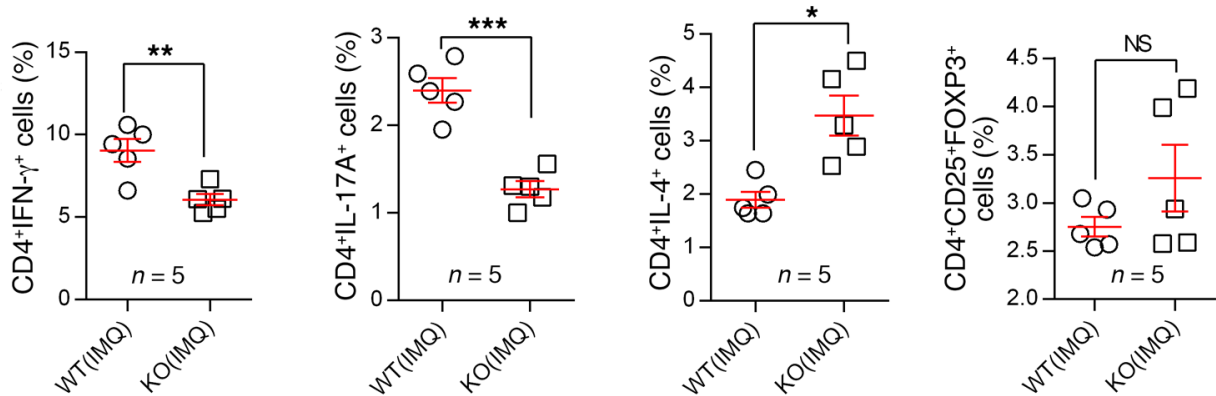

Figure 4. miR-210 deletion blocks psoriasis development. (A) miR-210 expression in splenic CD4+ $T$ cells (upper panel) or skin (lower panel) from KO $(n=5)$ and WT mice $(n=5)$. (B) Phenotypic presentation and H\&E staining of mouse back skin for WT or KO mice treated with IMQ or vehicle for 7 days. Scale bars: $100 \mu \mathrm{m}$ (C and D) Splenomegaly (C), acanthosis (D, left panel), and dermal cellular infiltration (D, right panel) in WT or KO mice treated with IMQ $(n=5)$ or vehicle $(n=5)$ for 7 days. (E) Representative flow cytometric analysis of Th1, Th2, Th17, and Treg cells in splenic CD4+ T cells from WT $(n=5)$ or KO $(n=5)$ mice treated with IMQ for 7 days. Statistical data are shown in the right panel. All data are obtained from miR-210 KO or WT mice with C57BL/6) background and are representative of at least 3 independent experiments with 5 samples per group in each. Data represent the mean \pm SEM. ${ }^{*} P<0.05$, ${ }^{* *} P<0.01$, ${ }^{* * *} P<0.001$. NS, not significant. Two-tailed Mann-Whitney $U$ test (A), 1-way ANOVA with Bonferroni's post hoc test (D), or 2-tailed unpaired Student's $t$ test (E) was used.

by antagomir-210 markedly increased the percentage of Th2 cells and decreased the percentage of Th1 and Th17 cells in splenic cells of IMQ-treated mice (Figure 5G). In addition, IMQ-treated mice injected with antagomir-210 expressed lower levels of Il17a, Il17f, and Ifng but higher levels of Il4 mRNA in both skin lesions and splenic $\mathrm{CD} 4^{+} \mathrm{T}$ cells (Figure $5 \mathrm{H}$ ). Taken together, these results indicated that the inhibition of miR-210 expression in vivo can prevent the immunopathological changes in psoriasis and can effectively ameliorate disease severity, suggesting a novel treatment strategy for psoriasis. 
A

Antagomir-210 or antagomir-NC

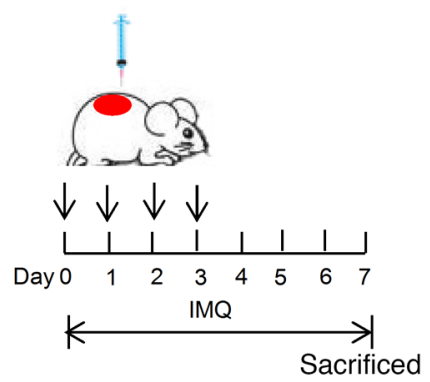

C Vehicle
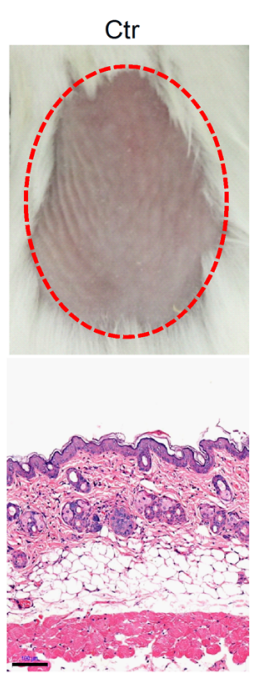

G
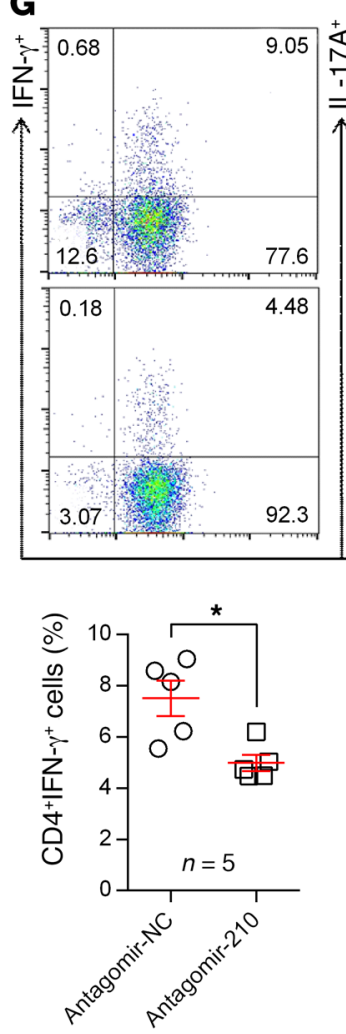

B

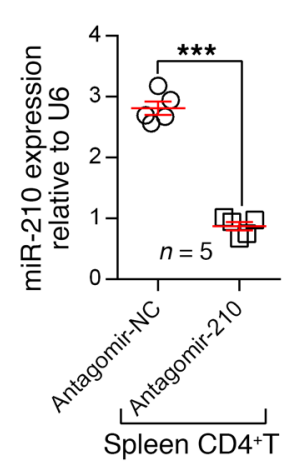

IMQ
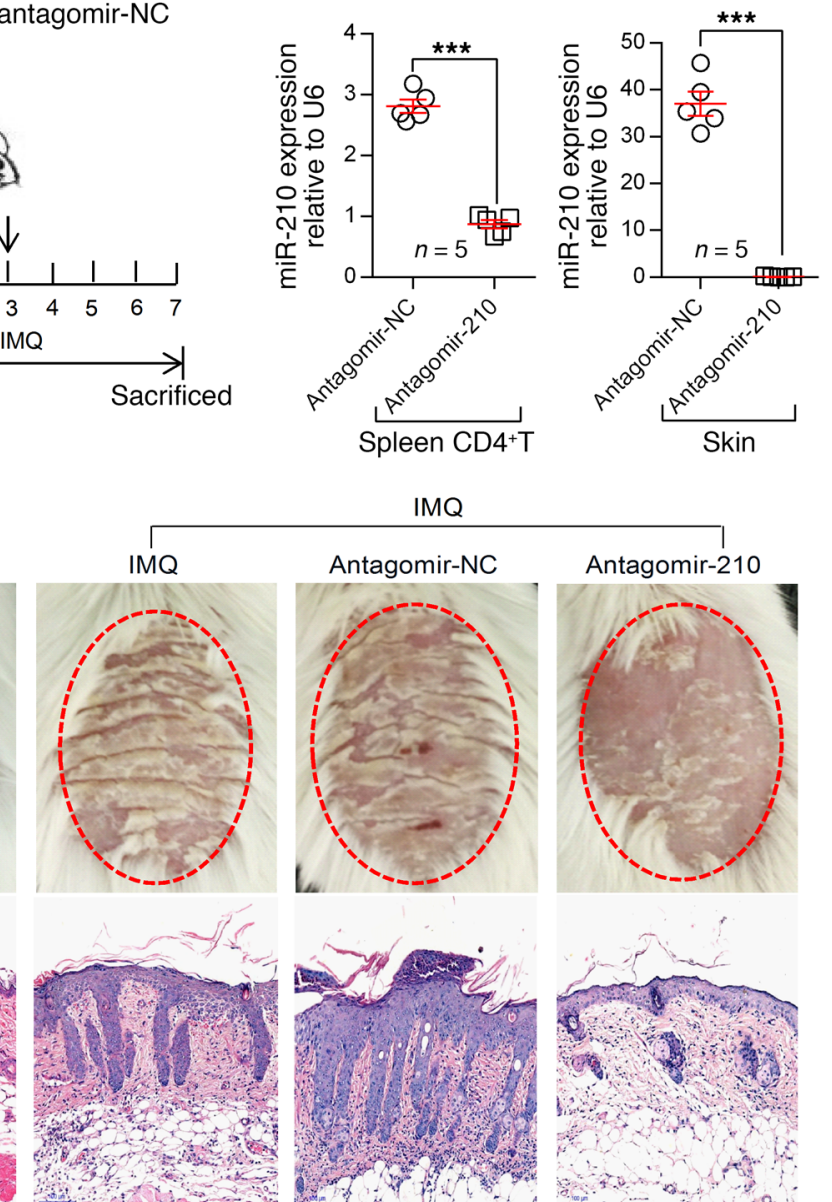

D

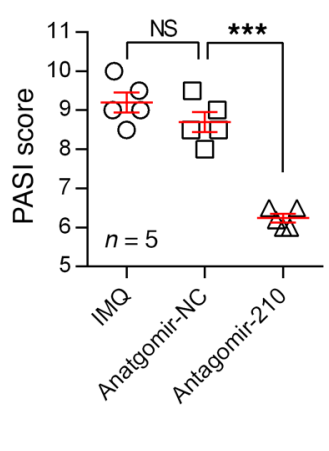

E
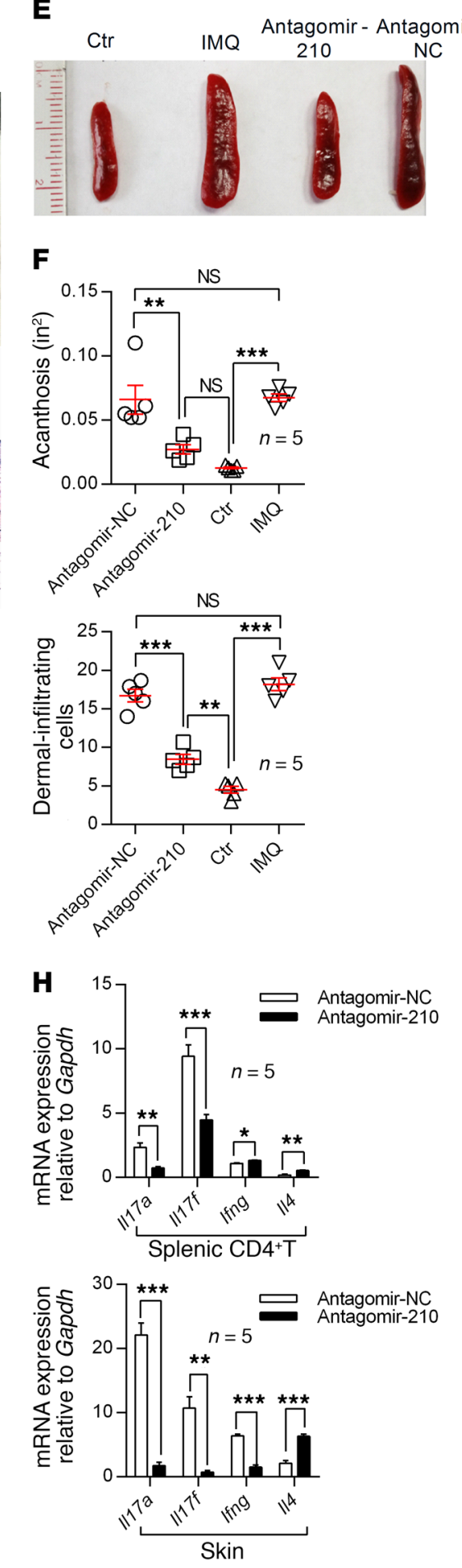
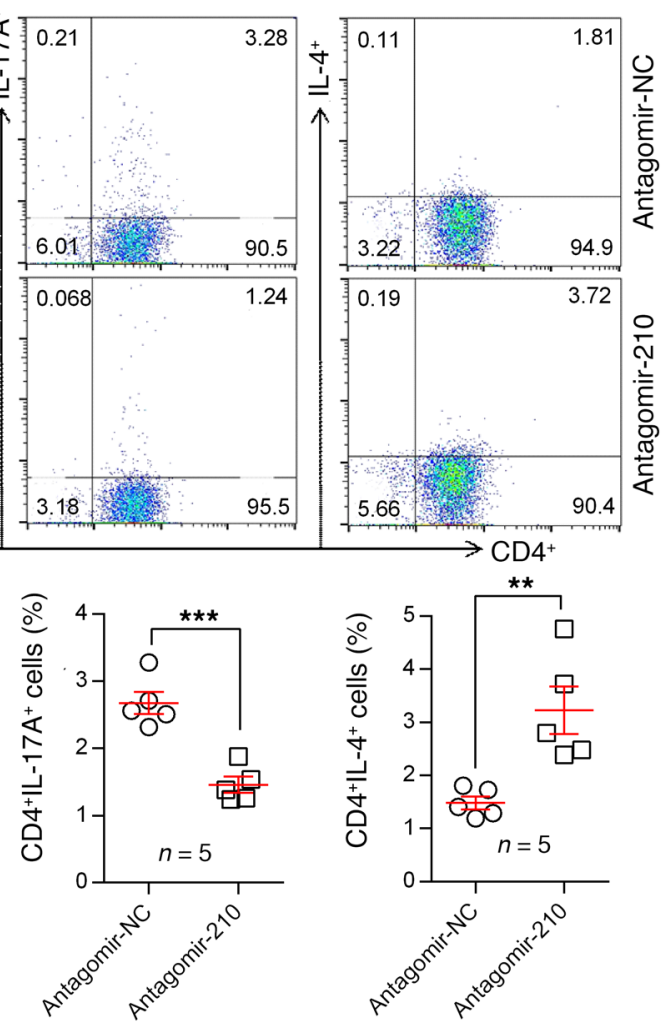

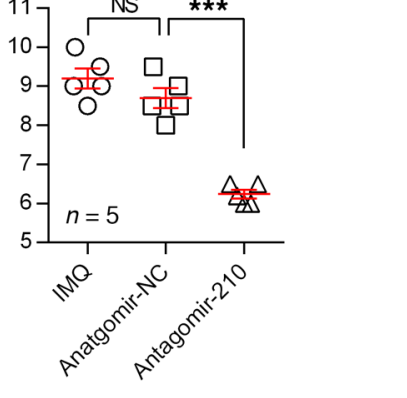
NC
NC

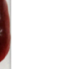


Figure 5. Intradermal administration of antagomir-210 ameliorates the pathological phenotype of IMQ-induced psoriasis-like mice. (A) Schematic diagram for intradermal administration of antagomir-NC (10 nmol) or antagomir-210 (10 nmol) on day 0, 1, 2, and 3 during the application of IMQ in BALB/c mice. (B) miR-210 expression in splenic CD4 ${ }^{+}$T cells (left panel) or skin lesions (right panel) from antagomir-NC-treated $(n=5)$ or antagomir-210-treated mice $(n=5)$. (C) The clinical manifestations and H\&E staining of the back skin derived from mice injected with antagomirNC or antagomir-210. Scale bars: $100 \mu \mathrm{m}$. (D) PASI score of mice in $\mathbf{A}$ $(n=5)$. (E) Splenomegaly of mice in $\mathbf{A}$. (F) Acanthosis (upper panel) and dermal cellular infiltrates (lower panel) were quantified for control mice $(n=5)$ and IMQ-induced mice $(n=5)$ treated with antagomir-NC $(n=5)$ or antagomir-210 $(n=5)$. (G) Representative flow cytometric analysis of Th1, Th17, and Th2 cells in splenic CD4+ ${ }^{+}$cells from antagomir-NC-treated $(n=5)$ and antagomir-210-treated IMQ mice $(n=5)$. Statistical data are shown in the lower panel. (H) The mRNA expression of II17a, II17f, Ifng, and $1 / 4$ in splenic CD4 ${ }^{+}$T cells (upper panel) or skin lesions (lower panel) from mice treated with antagomir-NC $(n=5)$ or antagomir-210 $(n=5)$. All results are representative of at least 3 independent experiments with 3 to 5 samples per group in each. Data represent the mean \pm SEM. ${ }^{*} P<0.05,{ }^{*} P<0.01$, ${ }^{* *} P<0.001$. NS, not significant. Two-tailed unpaired Student's $t$ test (B, G, and $\mathbf{H}$ ) or 1-way ANOVA with Bonferroni's post hoc test ( $\mathbf{D}$ and $\mathbf{F}$ ) was used.

STAT6 and LYN are downstream targets of miR-210. To gain insight into the molecular mechanisms by which miR-210 regulates Th cell differentiation in psoriasis, we combined 4 bioinformatic tools, TargetScan, miRWalk, miRanda, and RNA22, to predict the putative targets of miR-210 (Supplemental Table 3). Among them, immune-related genes were of special interest because our results suggested a role for miR-210 in regulating $\mathrm{Th}$ cell differentiation. Two potential target genes, STAT6 and LYN, which have been reported to regulate $\mathrm{T}$ cell function, were selected for this study. To validate these 2 target genes, we generated WT luciferase reporter constructs that included the $3^{\prime}$ untranslated region (UTR) of the STAT6 and LYN genes and mutant type (MU) reporter constructs, which contained mutant binding sequences of miR-210 (Figure 6A). Compared with the agomir-NC-transfected cells, cells transfected with agomir-210 showed significantly decreased luciferase activity with the WT constructs, but this inhibition was lost with transfection of the MU construct (Figure 6B). In mammalian cells, the majority of mature miRs are loaded onto argonaute 2 (Ago2) proteins to generate the effector complex, RNAinduced silencing complex (RISC), through which target mRNA degradation or transcription inhibition is mediated (27). To further confirm whether STAT6 and LYN are downstream targets of miR-210, we assayed Ago2 immunoprecipitates of $\mathrm{CD}^{+} \mathrm{T}$ cells derived from psoriasis patients and normal controls. As expected, the mRNA levels of miR-210, STAT6, and LYN detected in psoriatic $\mathrm{CD}^{+} \mathrm{T}$ cell immunoprecipitates were much higher than those in normal CD $4^{+} \mathrm{T}$ cells (Supplemental Figure 13A). Correspondingly, as a technique control, we found a 232.2-fold increase in U1 expression level in the SNRNP70 immunoprecipitates compared with the IgG control (Supplemental Figure 13B).

We next sought to evaluate whether miR-210 influences endogenous STAT6 and LYN protein levels. First, psoriatic CD4 ${ }^{+} \mathrm{T}$ cells were transfected with antagomir-210, and normal CD $4^{+} \mathrm{T}$ cells were transfected with agomir-210. Notably, we found that the protein levels of both STAT6 and LYN in CD4 ${ }^{+}$T cells were decreased by transfection with agomir-210 but were significantly increased after transfection with antagomir-210 compared with their corresponding controls (Figure 6C and Supplemental Figure 14, A and B). Strikingly, the protein and mRNA levels of the 2 targets were dramatically increased in splenic CD4 ${ }^{+} \mathrm{T}$ cells from IMQ-treated $\mathrm{KO}$ mice compared with those from IMQ-treated WT mice (Figure 6D and Supplemental Figure 14, C-F). In addition, we also found that the 2 targets of miR-210 were significantly downregulated at both the protein and the mRNA levels in psoriatic $\mathrm{CD} 4^{+} \mathrm{T}$ cells from patients and IMQ-treated mice compared with normal controls (Figure 6, $\mathrm{E}$ and F, and Supplemental Figure 15, A-E). Moreover, the mRNA levels of STAT6 and $L Y N$ were negatively correlated with miR-210 expression in $\mathrm{CD}^{+} \mathrm{T}$ cells from psoriasis patients (Supplemental Figure 15F). Consistent with their expression in CD $4^{+}$T cells, STAT6 and LYN protein and mRNA levels were also significantly decreased in lesional skins from both psoriasis patients and IMQ-treated mice (Figure 6, G and H, and Supplemental Figure 16). Together, these data from luciferase reporter assay, RNA immunoprecipitate assay, and transfection experiments indicated that STAT6 and LYN are direct targets of miR-210.

To explore the biological role and the importance of STAT6 and LYN in immune regulation, we performed gain- and lossof-function assays of normal CD4 ${ }^{+} \mathrm{T}$ cells activated by anti-CD3 and anti-CD28 Abs via transfection with STAT6 or LYN siRNAs, as well as their overexpression vectors (Figure 7, A and B). The results from both knockdown and overexpression experiments indicated that STAT6 and LYN inhibited IL17A, IL17F, and IFNG mRNA expression and promoted IL4 expression (Figure 7, A and B). We next performed gene recovery experiments in $\mathrm{CD}^{+} \mathrm{T}$ cells with miR-210 overexpression. Strikingly, overexpression of either STAT6 or LYN partly abolished the regulatory effects of miR-210 on cytokine expression (Figure 7, C and D). Our data indicated that miR-210 regulates $\mathrm{T}$ cell cytokine expression through repressing its targets STAT6 and LYN.

HIF-1 $\alpha$ mediates TGF- $\beta$ - and IL-23-induced miR-210 overexpression via epigenetic regulation. To identify the regulatory sequence critical for miR-210 transcription, we established 3 reporter constructs that included different fragments of miR-210 promoter regions upstream of the transcription start site (TSS) (fragment S1: from $-1,000$ bp to $-501 \mathrm{bp}$; S2: from $-500 \mathrm{bp}$ to $-1 \mathrm{bp}$; S3: from $-1,000$ bp to $-1 \mathrm{bp}$ ) (Supplemental Figure 17A). These reporter constructs were transfected into HEK293T cells separately, and luciferase activity was measured. The results indicated that the S3 reporter construct had the highest luciferase activity in transfected HEK293T cells, and the effects of S2 on assay activity were stronger than those of S1 (Supplemental Figure 17B), suggesting that the core promoter region was included within $-500 \mathrm{bp}$ upstream of the TSS of miR-210. A previous study showed that HIF-1 $\alpha$ regulated miR-210 expression in many tumors (28). We also found a potential HIF-1 $\alpha$ binding sequence in the S2 fragment (from -420 to $-436 \mathrm{bp}$ ) (Supplemental Figure 17C), suggesting that miR-210 expression is regulated by HIF-1 $\alpha$ in $\mathrm{CD} 4^{+} \mathrm{T}$ cells of psoriasis. We generated reporter constructs with WT and mutated HIF-1 $\alpha$ binding site in S2 (HIF- $1 \alpha$ WT and HIF- $1 \alpha$ MU) and found strikingly decreased luciferase activity in HEK293T cells transfected with HIF-1 $\alpha$ MU compared with HEK293T cells transfected with HIF-1 $\alpha$ WT (Supplemental Figure 17D). In contrast to HIF-1 $\alpha$ MU, HIF-1 $\alpha$ overexpression led to significantly 
A

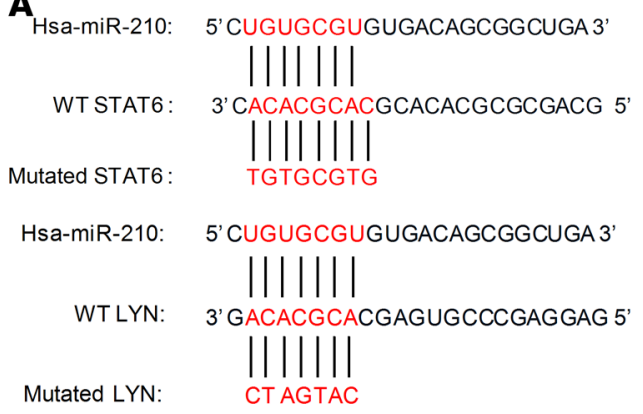

B

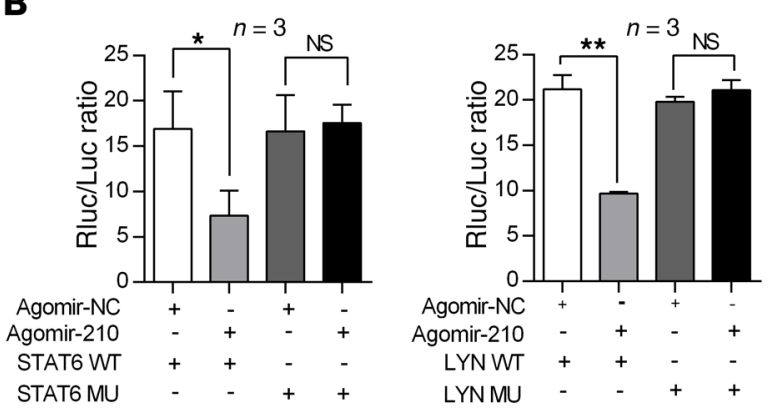

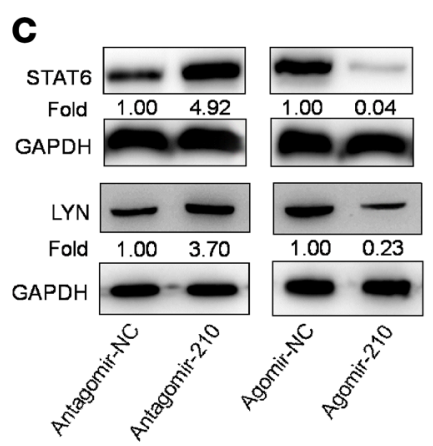

$\mathbf{F}$

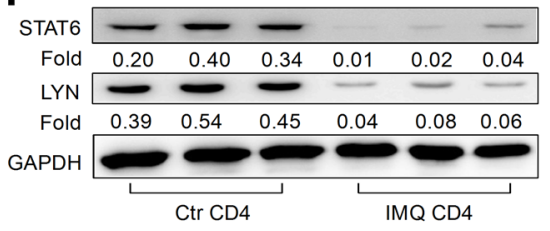

D

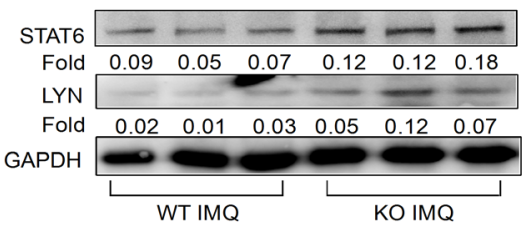

E

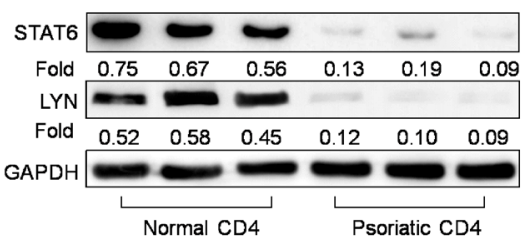

G

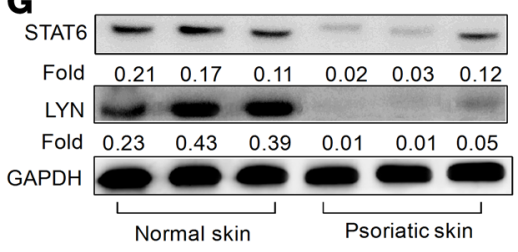

H

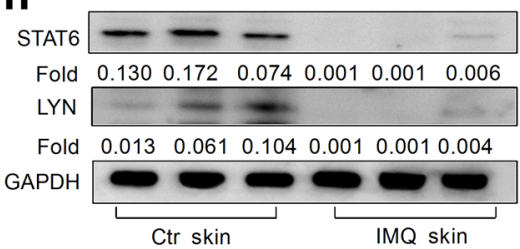

Figure 6. STAT6 and LYN are downstream targets of miR-210. (A) miR-210 target sequences (in red) of the 3'-UTR of STAT6 (A, upper panel) and LYN (A, lower panel) mRNAs and corresponding mutant sequences, which were included in luciferase reporter vectors. (B) Luciferase activities were measured in HEK293T cells cotransfected with luciferase reporter vectors and agomir-210 or agomir-NC, $n=3$ per group. (C) Normal CD4 ${ }^{+}$T cells were transfected with agomir-210 or agomir-NC, and psoriatic CD4+ T cells were transfected with antagomir-210 or antagomir-NC. STAT6 (upper panel) and LYN (lower panel) protein levels were analyzed. (D) The protein levels of STAT6 and LYN in splenic CD4+ T cells from IMQ-treated WT and KO mice. (E-H) STAT6 and LYN protein levels in $\mathrm{CD}^{+} \mathrm{T}$ cells $(\mathbf{E}$ and $\mathbf{F})$ and skin lesions $(\mathbf{G}$ and $\mathbf{H})$ derived from psoriasis patients and IMQ-induced psoriasis-like mouse models (BALB/C) as well as their healthy controls. Data $\left(\mathbf{B}\right.$ and $\mathbf{C}$ ) are representative of at least 3 independent experiments. Data represent the mean $\pm \mathrm{SEM}$. ${ }^{*} P<0.05,{ }^{* *} P<0.01$. NS, not significant. Two-tailed unpaired Student's $t$ test (B) was used.

increased luciferase activity in HIF-1 $\alpha$ WT-transfected HEK293T cells (Supplemental Figure 17E). In addition, we also performed chromatin immunoprecipitation (ChIP) assays and confirmed that HIF- $1 \alpha$ bound to the S2 region in the miR-210 promoter region (Supplemental Figure 17F). To further confirm that HIF-1 $\alpha$ regulates miR-210 expression in psoriasis, we determined HIF-1 $\alpha$ expression in psoriatic $\mathrm{CD}^{+} \mathrm{T}$ cells and skin. As expected, HIF-1 $\alpha$ protein levels were significantly elevated in psoriatic $\mathrm{CD}^{+} \mathrm{T}$ cells and skin lesions derived from both psoriasis patients and IMQ-treated mice (Supplemental Figure 18, A-D). In addition, the protein level of HIF-1 $\alpha$ was positively related with miR210 expression in psoriatic $\mathrm{CD}^{+}{ }^{+} \mathrm{T}$ cells (Supplemental Figure 18E). When HIF-1 $\alpha$ siRNA, HIF-1 $\alpha$ overexpression plasmid, and their corresponding controls were transfected into psoriatic CD $4^{+}$ $\mathrm{T}$ cells or normal $\mathrm{CD} 4^{+} \mathrm{T}$ cells, miR-210 expression was inhibited in psoriatic $\mathrm{CD}^{+} \mathrm{T}$ cells transfected with HIF- $1 \alpha$ siRNA but was promoted in normal CD $4^{+} \mathrm{T}$ cells transfected with the HIF-1 $\alpha$ overexpression plasmid (Supplemental Figure 19). These results indicated that HIF- $1 \alpha$ binds to the miR-210 promoter region and promotes miR-210 expression.

To demonstrate the upstream mechanism of miR-210 overexpression in $\mathrm{CD}^{+} \mathrm{T}$ cells and skin lesions of psoriasis patients, we investigated whether some inflammatory cytokines including IL-23, TGF- $\beta$, IL-6, and IL-1 $\beta$, which are very important for Th17 cell differentiation and are increased in psoriasis (29), induced miR-210 expression in CD $4^{+} \mathrm{T}$ cells. We stimulated CD $4^{+} \mathrm{T}$ cells with these cytokines individually and found that TGF- $\beta$ or IL-23 stimulation, but not IL- 6 or IL-1 $\beta$, can increase the expression of miR-210 significantly, among which TGF- $\beta$ induced the greatest increase of miR-210 (Figure 8A). Furthermore, we investigated whether the induction of miR-210 by TGF- $\beta$ and IL-23 was mediated by HIF- $1 \alpha$. We stimulated CD $4^{+}$T cells with TGF- $\beta$ and IL-23 individually and found an increased HIF-1 $\alpha$ protein level together with decreased STAT6 and LYN protein levels, which showed time-dependent changes (Figure 8B and Supplemental Figure 20). Moreover, when HIF-1 $\alpha$ expression was inhibited by 
A
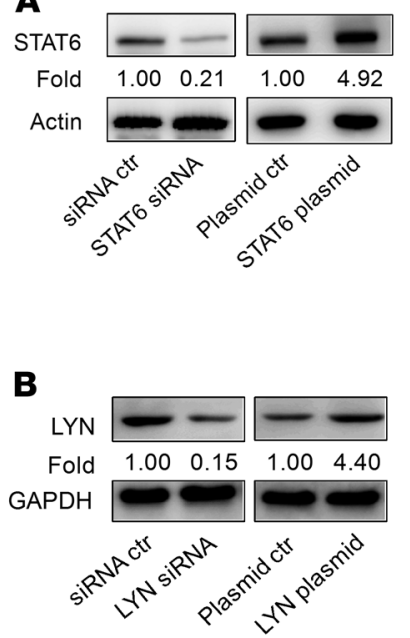

C
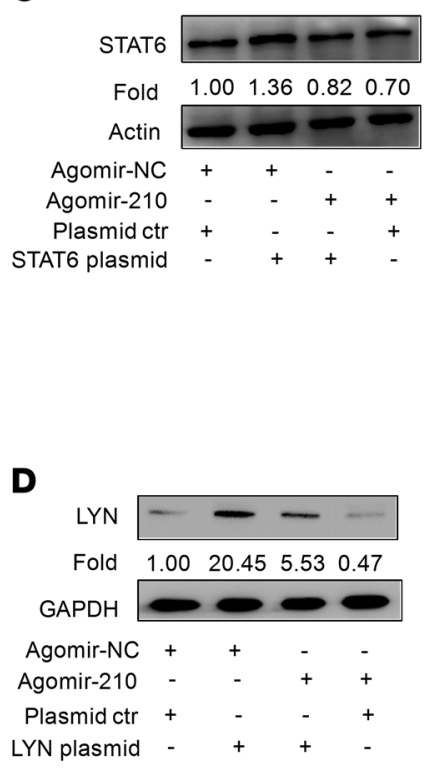
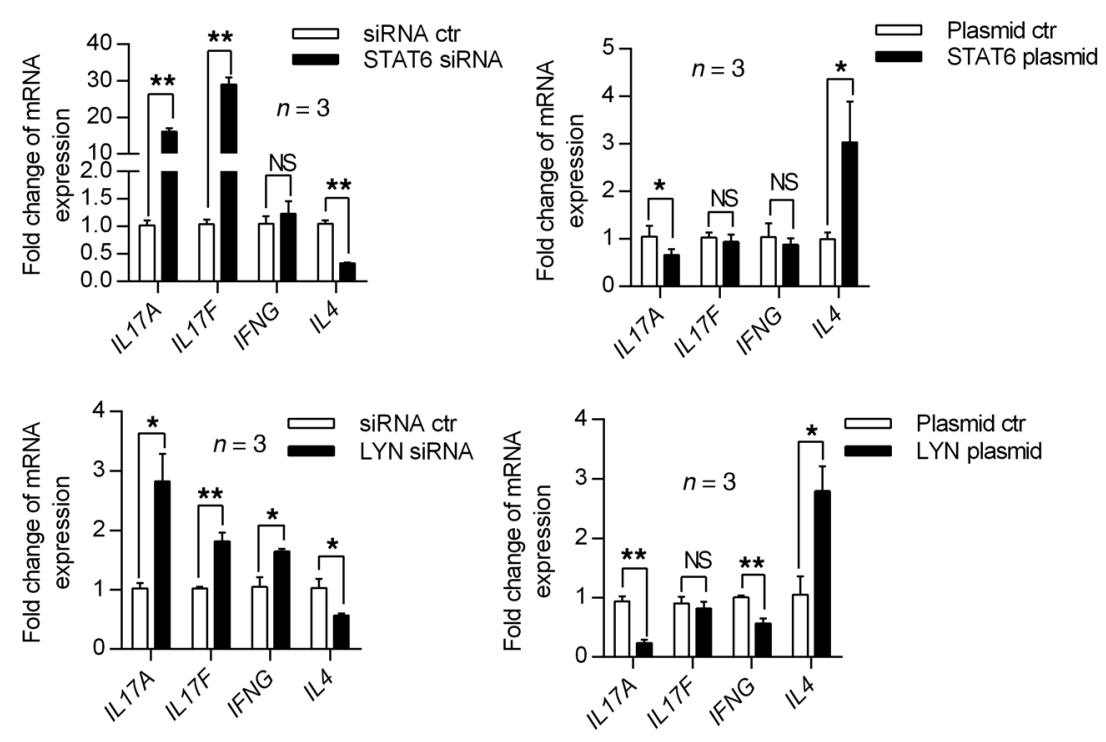

Figure 7. STAT6 and LYN are required for miR-210-induced effects. (A and B) Normal human $\mathrm{CD} 4^{+} \mathrm{T}$ cells were transfected with STAT6 siRNAs and overexpression plasmid $(\mathbf{A}, n=3)$ or LYN siRNAs and overexpression plasmid (B, $n=3$ ) separately for 48 hours. STAT6 and LYN protein levels (left panel), and the mRNA levels of IL17A, IL17F, IFNG, and IL4 (middle and right panels) were detected in transfected cells. (C and D) Normal human $\mathrm{CD} 4^{+} \mathrm{T}$ cells were transfected with agomir-210 or agomir-NC, and after 24 hours, the STAT6 overexpression plasmid or plasmid control $(\mathbf{C}, n=3)$ and LYN overexpression plasmid or plasmid control (D, $n=3$ ) were transfected into cells. Then, cells were collected to detect the protein levels of STAT6 or LYN (left panel) and the mRNA levels of IL17A, IL17F, IFNG, and IL4 (right panel) at 48 hours after transfection. Similar results were obtained from 3 independent experiments. Data represent the mean $\pm \mathrm{SEM} .{ }^{*} P$ $<0.05$, ${ }^{* *} P<0.01,{ }^{* *} P<0.001$. NS, not significant. Two-tailed unpaired Student's $t$ test (A and $\mathbf{B}$ ) or 1-way ANOVA with Bonferroni's post hoc test (C and $\mathbf{D})$ was used.
RNA interference, the induction of miR-210 by TGF- $\beta$ and IL- 23 was significantly weakened (Figure 8C). These data suggested that TGF- $\beta$ - and IL-23-induced upregulation of miR-210 was mediated by HIF- $1 \alpha$.

A series of studies have demonstrated that miR expression is regulated by epigenetic mechanisms (30-33). P300, a histone acetyltransferase, was shown to act as a transcriptional coactivator of HIF-1 $\alpha$, resulting in transcriptional activation of multiple genes through increasing histone acetylation in gene loci $(34,35)$. Thus, we investigated whether HIF-1 $\alpha$ regulates miR-210 expression via recruitment of P300. We first performed coimmunoprecipitation (Co-IP) experiments and confirmed that HIF-1 $\alpha$ and P300 formed a protein complex, which was increased in $\mathrm{CD} 4^{+} \mathrm{T}$ cells with TGF- $\beta$ or IL-23 stimulation (Figure 8D and Supplemental Figure 21). In addition, the miR-210 expression in $\mathrm{CD} 4^{+} \mathrm{T}$ cells transfected with
P300 siRNA was significantly decreased, indicating that P300 has a similar effect to that of HIF-1 $\alpha$ on miR-210 expression (Supplemental Figure 22). We next compared histone $\mathrm{H} 3$ acetylation (H3ac) levels on the miR-210 promoter in $\mathrm{CD}^{+} \mathrm{T}$ cells derived from psoriasis patients and normal controls. Notably, psoriatic $\mathrm{CD} 4^{+} \mathrm{T}$ cells exhibited increased $\mathrm{H} 3 \mathrm{ac}$ levels on the miR-210 promoter compared with normal $\mathrm{CD} 4^{+} \mathrm{T}$ cells (Supplemental Figure 23). To determine whether increased $\mathrm{H} 3 \mathrm{ac}$ on the miR-210 promoter is mediated by the HIF-1 $\alpha / \mathrm{P} 300$ complex, we transfected psoriatic $\mathrm{CD} 4^{+}$ $\mathrm{T}$ cells with HIF- $1 \alpha$ siRNA and normal $\mathrm{CD} 4^{+} \mathrm{T}$ cells with HIF- $1 \alpha$ plasmid for ChIP assays. The results showed that the enrichment of HIF-1 $\alpha$, P300, and H3ac levels on the miR-210 promoter were suppressed in psoriatic $\mathrm{CD} 4^{+} \mathrm{T}$ cells by HIF-1 $\alpha$ siRNA, but were notably increased in normal $\mathrm{CD} 4^{+} \mathrm{T}$ cells by the HIF-1 $\alpha$ plasmid (Figure 8, $\mathrm{E}$ and F). Moreover, TGF- $\beta$ or IL-23 stimulation also promoted the 
A
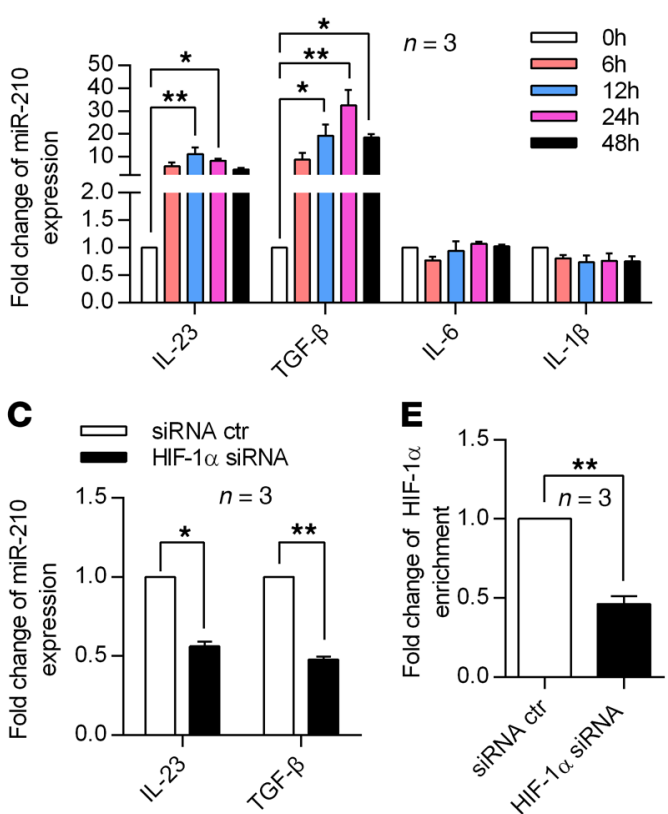

D

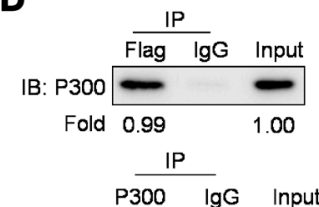

IB: HIF-1 $\rightleftharpoons \mathrm{Ctr}$

Fold $0.20 \quad 1.00$

IB: HIF-1a $\longrightarrow$ TGF-

Fold $0.49 \quad 1.00$

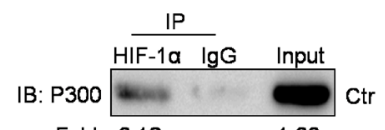

Fold $0.12 \quad 1.00$

IB: P300 TGF- $\beta$

Fold 0.41
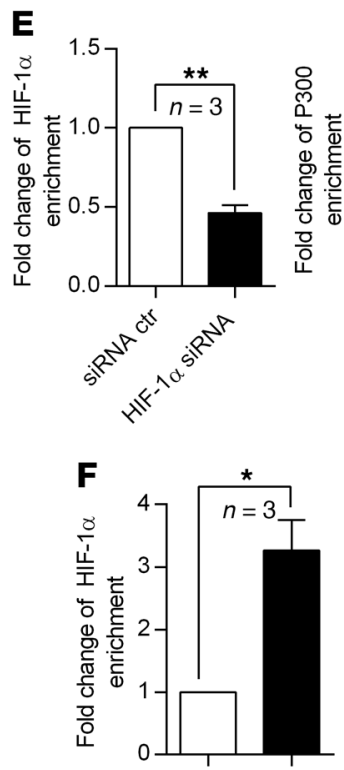
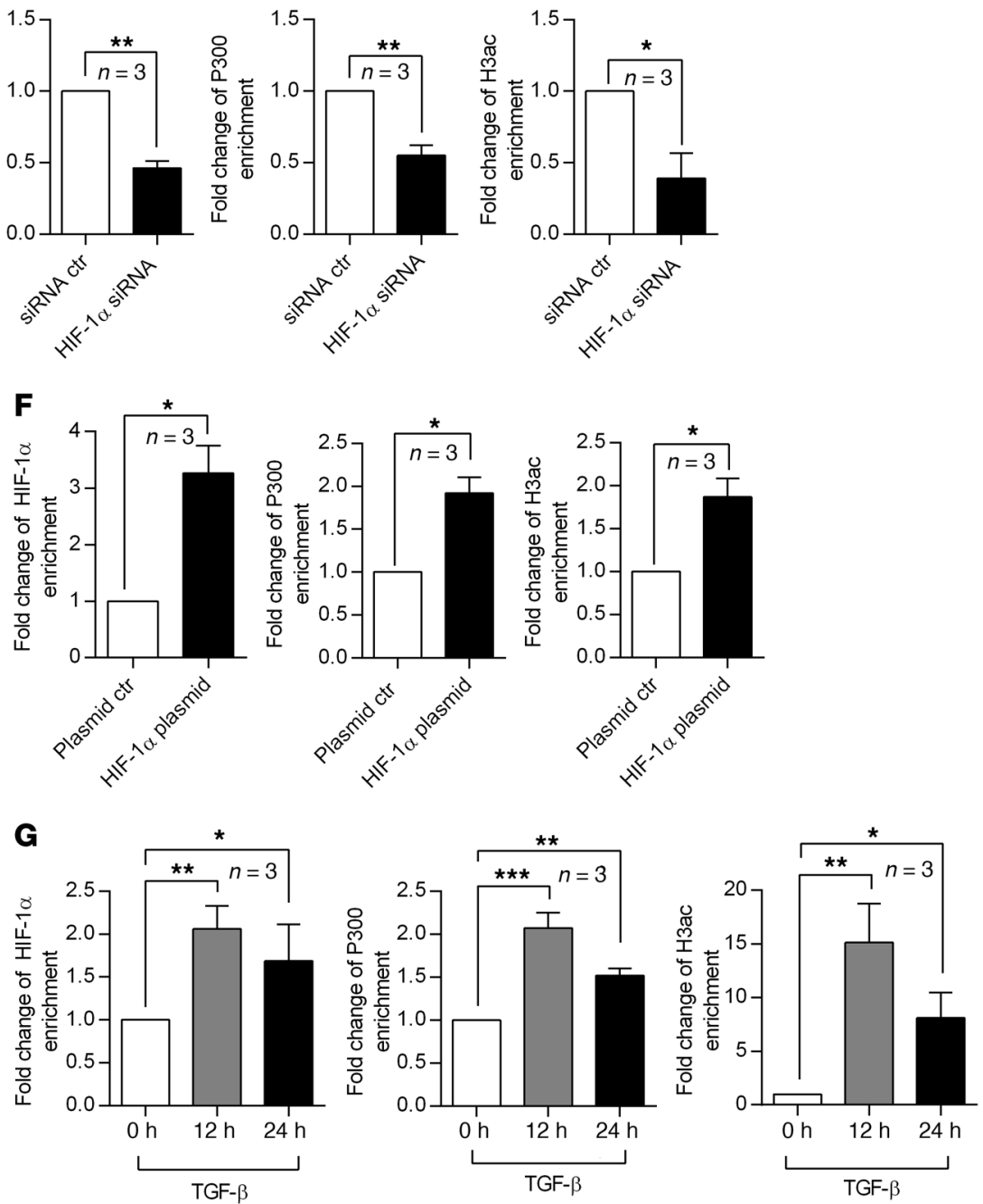

Figure 8. HIF-1 $\alpha$ mediates TGF- $\boldsymbol{\beta}$ - and IL-23-induced miR-210 overexpression via epigenetic regulation. (A and B) The expression of miR-210 in normal human CD4+ ${ }^{+}$cells stimulated with IL-23, TCF- $\beta$, IL-6, or IL-1 $\beta$ (A, $n=3$ per group). The protein levels of HIF- $1 \alpha$, STAT6, and LYN in cells stimulated with IL-23 or TCF- $\beta$ (B). (C) The miR-210 expression levels in human CD4+ T cells transfected with HIF-1 $\alpha$ siRNA or siRNA controls followed by stimulation with IL-23 or TGF- $\beta$ for 24 hours ( $n=3$ per group). (D) CD4+ T cells were transfected with HIF-1 $\alpha$ overexpression plasmid (upper panel) or were stimulated with TGF- $\beta$ (lower 2 panels). Co-IP and Western blot showed the interaction between HIF-1 $\alpha$ and P300. (E-G) The enrichment of HIF-1 $\alpha$, P300, and H3ac on the miR-210 promoter in psoriatic CD4+ T cells transfected with HIF-1 $\alpha$ siRNA or controls (E, $n=3)$, normal CD4+ $4^{+}$cells transfected with HIF-1 $\alpha$ plasmid or controls (F, $n=3$ ), or normal $\mathrm{CD}^{+}$T cells stimulated with TGF- $\beta(\mathbf{G}, n=3)$. Data $(\mathbf{B}$ and $\mathbf{D})$ are representative of at least 3 independent experiments. Data represent the mean \pm SEM. ${ }^{*} P<0.05,{ }^{* *} P<0.01,{ }^{* *} P<0.001$. One-way ANOVA with Dunnett's post hoc test (A and $\left.\mathbf{G}\right)$ or 2 -tailed unpaired Student's $t$ test (C, E, and F) was used.

enrichment of HIF-1 $\alpha, \mathrm{P} 300$, and $\mathrm{H} 3 \mathrm{ac}$ on the miR-210 promoter (Figure $8 \mathrm{G}$ and Supplemental Figure 24). These data indicated that TGF- $\beta$ and IL-23 could induce miR-210 expression through HIF- $1 \alpha$ / P300-mediated epigenetic mechanisms in psoriasis.

\section{Discussion}

Accumulating evidence has revealed that miRs are frequently involved in regulating immune cell development, differentiation, and immune responses (36-38). Here, we showed that miR-210 


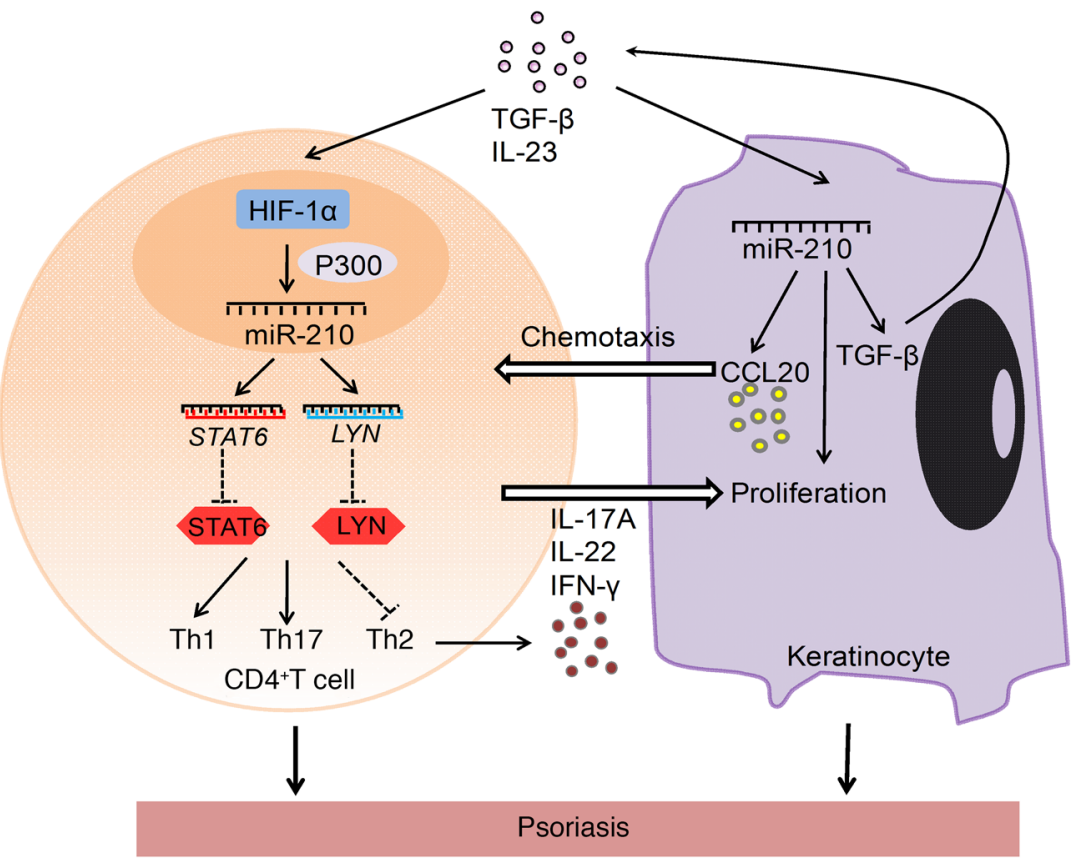

Figure 9. Schematic illustration of TGF- $\beta /$ IL-23-HIF$1 \alpha$-miR-210-STAT6/LYN pathways contributing to the pathogenesis of psoriasis. In psoriasis patients, inflammatory factors TGF- $\beta$ and IL-23 stimulate the expression of HIF-1 $\alpha$, which recruits $\mathrm{P} 300$ to the miR-210 promoter region and increases the $\mathrm{H} 3 \mathrm{ac}$ levels on miR-210 promoters, thus leading to the upregulation of miR-210 expression. miR-210 represses STAT6 and LYN expression through binding the $3^{\prime}$-UTRs of STAT6 and LYN mRNA, which promotes Th1/Th17 cell differentiation and inhibits Th2 cell differentiation in psoriasis. Moreover, miR210 overexpression also enhances the proliferation and chemokine secretion of keratinocytes, which increases the recruitment of activated T cells in skin lesions. Together, the effects of the increased miR210 in $\mathrm{CD}^{+} \mathrm{T}$ cells and keratinocytes contribute to the formation of psoriatic skin lesions. is upregulated in psoriatic CD $4^{+} \mathrm{T}$ cells and skin lesions and contributes to the immune imbalance of psoriasis by promoting the differentiation of Th17 and Th1 cells and inhibiting Th2 cell differentiation in vitro and in vivo. Inhibition of miR-210 prevents the formation of skin lesions in a psoriasis-like mouse model. In addition, we investigated the in vivo effect of miR-210 on Th22 cell response, which has also been reported to be involved in the pathogenesis of psoriasis $(6,7,39)$. We detected the expression of Th22-related genes including Il22, Ahr, Ccr4, Ccr6, and Ccr1O in mouse splenic $\mathrm{CD} 4^{+} \mathrm{T}$ cells from IMQ-induced mice injected with agomir-210/agomir-NC or antagomir-210/antagomir-NC. However, no significant change was observed in the expression levels of these genes in the agomir-210 or antagomir-210 group compared with the agomir-NC or antagomir-NC group (Supplemental Figure 25). The results suggested that overexpressed miR-210 contributes to psoriasis through inducing Th17 and Th1 cells, but not Th22.

A miR often interacts with hundreds of target mRNAs; however, only a small number of key target genes mediate its function (13). A previous study reported that miR-210 substantially inhibited the differentiation of Th17 cells under reoxygenation due to a negativefeedback mechanism by miR-210 to inhibit protein expression of its target HIF-1 $\alpha$ (40). However, in psoriasis, cell proliferation and inflammatory responses increase oxygen consumption, and epidermal thickening leads to impaired oxygen supply, resulting in intensified hypoxia and consequent HIF-1 $\alpha$ activation (41). Consistent with these observations, our studies demonstrated that both HIF-1 $\alpha$ and miR-210 expression, 2 hypoxia-induced factors, were significantly elevated in psoriatic skins and $\mathrm{CD} 4^{+} \mathrm{T}$ cells and that miR210 promotes Th17 differentiation both under reoxygenation and normoxia (Supplemental Figure 26), suggesting that HIF-1 $\alpha$ is not a downstream target of miR-210 in $\mathrm{CD}^{+} \mathrm{T}$ cells of psoriasis. This discrepancy may be due to the specific tissue microenviroment and complex pathogenesis of psoriasis. Actually, we identified STAT6 and LYN as functional targets of miR-210 in the present study, which mediate the regulatory effects of miR-210 on Th cell differentiation. STAT6 is a well-known key mediator of Th2 differentiation that integrates signaling from the IL-4/IL-13 receptor complex (42). Stat $6^{-/-}$mice exhibit strengthened Th1 and Th17 immune responses, expressing sufficient Tbx21 and Rorc genes (43). LYN is a cytoplasmic membrane-associated tyrosine kinase that regulates various cellular processes through phosphorylation of inhibitory receptors, enzymes, and adaptors (44). $\mathrm{Lyn}^{-/-}$mice can develop a disease reminiscent of systemic lupus erythematosus $(44,45)$ but also show simultaneous enhancement of Th2 immunity $(42,46$, 47). Interestingly, Stat $^{-/-} \mathrm{Lyn}^{-/-}$mice were reported to have reduced Th2 traits in young mice and early-onset autoimmune disease (42). This previous evidence supported our finding that miR-210 overexpression induced Th17 and Th1 differentiation and inhibited Th2 differentiation through targeting STAT6 and LYN in psoriasis.

Proinflammatory cytokine stimulation is a key initiating factor for formation of psoriatic skin lesions. Previous studies showed that IL-23, TGF- $\beta$, IL-6, and IL-1 $\beta$, which are essential for Th17 cell differentiation, are increased in serum and skin lesions of psoriasis (29). However, the mechanism by which these inflammatory cytokines promote psoriasis is still unclear. Our previous study suggested that aberrant epigenetic modifications were involved in the pathogenesis of psoriasis (48). In this study, we found that TGF- $\beta$ and IL-23 can increase HIF- $1 \alpha$ expression and promote the interaction between HIF- $1 \alpha$ and P300 in $\mathrm{CD}^{+} \mathrm{T}$ cells. The increased HIF- $1 \alpha$ caused hyperacetylation of histone $\mathrm{H} 3$ in the miR-210 promoter region through recruitment of P300. Histone H3 hyperacetylation has been confirmed to be associated with transcription activation of genes (49). Consistently, we also found that the acetylation level of the miR-210 promoter was increased in $\mathrm{CD}^{+} \mathrm{T}$ cells of psoriasis patients. Taken together, these findings revealed a potentially novel upstream regulatory mechanism of excessive miR-210 expression in $\mathrm{CD}^{+} \mathrm{T}$ cells of psoriasis patients, in which TGF- $\beta$ - and IL-23-induced HIF-1 $\alpha$ upregulates 
miR-210 expression, which highlights an important role for miR210 in the proinflammatory factor-induced immune imbalance and skin lesion formation in psoriasis.

In addition to immune cells, keratinocytes also play an indispensable role in the pathogenesis of psoriasis. Activated by proinflammatory factors, keratinocytes exhibit excessive proliferation and secretion of chemokines, such as CCL2O and CXCL10, playing a crucial role in amplifying the immune response in psoriatic plaques $(7,50,51)$. Our studies found that miR-210 expression is also elevated in the epidermis from psoriatic skin of both psoriasis patients and IMQ-induced psoriasis-like mouse models. HaCaT cells stimulated with IL-23, TGF- $\beta$, IL-17A, or IFN- $\gamma$ have significantly increased miR-210 expression (Supplemental Figure $27 \mathrm{~A}$ ). In addition, both the BM chimeric mice (KO BM-WT) and the Rag2 $2^{-1-}$ mice transferred with $\mathrm{T}$ cells with miR-210 deletion displayed a slightly thickened epidermis and infiltration of inflammatory cells in skin lesion induced by IMQ, suggesting that miR-210 overexpression in keratinocytes also contributes to the pathogenesis of psoriasis. Furthermore, we found that miR-210 overexpression promoted the proliferation of normal human epidermal keratinocytes (NHEKs) (Supplemental Figure 27, B and C) as well as the secretion of chemokines by keratinocytes, such as CCL20, enhancing the chemotaxis of NHEKs to CD4+ $4^{+}$lymphocytes (Supplemental Figure 28, A-D). Interestingly, overexpression of miR-210 in NHEKs also increased the production of TGF- $\beta$, suggesting a potential positive feedback between miR-210 and TGF- $\beta$ in keratinocytes (Supplemental Figure 28, A and B). Together, these additional data suggested that miR-210 serves as an important regulator in the interaction between immune cells and keratinocytes in psoriatic skin lesions.

In conclusion, the present study provides evidence that miR210, as a downstream target of HIF- $1 \alpha$ and inflammatory cytokines TGF- $\beta$ and IL-23, promotes Th17 and Th1 cell differentiation and inhibits Th2 cell differentiation through targeting STAT6 and LYN, thereby causing the immune imbalance and inflammatory response in peripheral blood and skin lesions of psoriasis. Moreover, miR-210 also enhances the proliferation and chemokine secretion of keratinocytes, which further promotes the formation of psoriatic skin lesions (Figure 9). Together, our studies show that miR-210 plays an important role in the formation of skin lesions and immune imbalance in psoriasis and provide a potential target for psoriasis treatment.

\section{Methods}

Human subjects. A total of 63 psoriatic patients who were diagnosed with psoriasis vulgaris by pathologic examination were recruited from outpatient clinics in the Second Xiangya Hospital of Central South University. Psoriasis disease activity was assessed using PASI score. Patient information is shown in Supplemental Table 1. A total of 80 sex- and age-matched healthy controls were recruited from the medical staff at the Second Xiangya Hospital.

Mice. The C57BL/6J and BALB/c mice were purchased from Slack Company. BALB/c mice were used for establishing the IMQ-induced psoriasis-like model and C57BL/6J mice were used for the IL-23induced psoriasis-like model as well as in vitro $\mathrm{T}$ cell differentiation. Rag2-/ mice were purchased from the Shanghai Research Center For Model Organisms. The miR-210-KO mice were generated by Shang- hai Biomodel Organism Science \& Technology Development Co. Ltd. In brief, Cas 9 mRNA and gRNA were obtained by in vitro transcription, and then they were injected into fertilized eggs of C57BL/6J mice via the microinjection technique to generate FO generation mice. The FO generation mice with the gene mutant were backcrossed to C57BL/6J mice to obtain heterozygous F1 mice. The miR-210-KO (mir-210 $\left.0^{-/}\right)$mice were generated by self-crossing between heterozygous F1 mice. We determined the genotype of the mice by PCR analyses of genomic DNA isolated from mouse tails using the following primers: for FO and F1 mice, forward, 5'-GGCGGGCGGGGCGTGAT-3' and reverse, 5'-GGGCAAGACCTGGCTGAGTGATGT-3'; for mir-210 $^{-/-}$mice, forward, 5'-GGCGGGCGGGGCGTGAT-3' and reverse, 5'-GGGCAAGACCTGGCTGAGTGATGT-3'. Female mice at 6 to 8 weeks of age were used for all experiments. All mice were maintained in specific pathogen-free conditions.

IMQ-induced psoriasis-like mouse model. Female BALB/c or miR210-KO mice with C57BL/6J background (6-8 weeks of age) were kept under controlled conditions. The mice were treated with a daily topical dose of $62.5 \mathrm{mg}$ of IMQ cream (5\%) (Sichuan Med-shine Pharmaceutical, H20030128) on the shaved back for 7 consecutive days. Control mice were treated with the same dose of vehicle cream.

IL-23-induced psoriasis-like mouse model. An IL-23-mediated mouse model of psoriasis was created as previously described $(16,22)$. Generally, ears of C57BL/6J mice were injected intradermally with $1 \mu \mathrm{g}$ rmIL-23 (R\&D Systems) dissolved in $25 \mu \mathrm{l}$ PBS into one ear and $25 \mu \mathrm{l}$ PBS into the contralateral ear. Injections were used persistently every other day for a total of 8 days (days 1, 3, 5, and 7). On the eighth day, ears were analyzed by histopathology and in situ hybridization.

Antagomir and agomir treatment. Female BALB/c mice (6-8 weeks of age) were injected intradermally with antagomir-210 (10 nmol in $150 \mu \mathrm{l} \mathrm{PBS}$ ) or agomir-210 ( $5 \mathrm{nmol}$ in $150 \mu \mathrm{l} \mathrm{PBS})$, as well as their corresponding controls, on days $0,1,2$, and 3 during the application of IMQ. For antagomir administration, all mice were sacrificed on the seventh day. For agomir treatment, 3 to 6 mice in each group were sacrificed on days 4, 7, 10, and 14. Antagomir-210 and agomir-210 as well as their controls were synthesized by Guangzhou RiboBio Co., Ltd. The oligonucleotide sequences of antagomir-210 and agomir-210 are shown as follows: antagomir-210, 5'-UCAGCCGCUGUGUCACACGCACAG-3'; agomir-210, sense 5'-CUGUGCGUGUGACAGCGGCUGA-3' and antisense 5'-UCAGCCGCUGUGUCACACGCACAG-3'. Universal negative controls for both agomir and antagomir are based on the sequences of cel-miR-67 in Caenorhabditis elegans, which has been confirmed to have minimal sequence identity with miRs in human, mouse, and rat.

Scoring severity of skin inflammation in psoriasis-like mouse model. An objective scoring system was developed to score the severity of back skin inflammation in the IMQ-induced psoriasis-like mouse model, which was based on the clinical PASI for psoriasis patients (21). Erythema, scaling, and thickening were scored independently on a scale from 0 to 4 : 0 , none; 1 , slight; 2 , moderate; 3 , marked; 4 , very marked. The total score was obtained by accumulating the 3 index scores (score 0-12).

In situ hybridization. In situ hybridization was performed as previously described (52). Briefly, 6- $\mu$ m-thick frozen sections of IMQ-induced mouse psoriasiform specimens, psoriasis patients' lesional specimens, and control specimens from healthy mice and humans were used for experiments. After incubation in acetylation solution $(0.02 \mathrm{M} \mathrm{HCl}, 1.3 \%$ triethanolamine, and $0.25 \%$ acetic 
anhydride in diethyl pyrocarbonate-treated water) for 10 minutes at room temperature, sections were treated with proteinase $\mathrm{K}(5 \mu \mathrm{g}$ / $\mathrm{ml}$ ) in PBS for 5 minutes, washed, and prehybridized for 4 hours. Hybridization with hsa-miR-210-3p 5'-DIG- and 3'-DIG-labeled miRCURY LNA detection probe (Exiqon, catalog 612239-360) was performed overnight at $50^{\circ} \mathrm{C}$. Slides were then washed with $5 \times$ SSC buffer followed by incubation in $0.2 \times \mathrm{SSC}$ buffer for 1 hour at $60^{\circ} \mathrm{C}$. The probe binding was detected by incubating sections with alkaline phosphatase-conjugated anti-DIG Ab (1:1,000; Roche, catalog $11093274910)$ overnight at $4^{\circ} \mathrm{C}$. Sections were visualized with a mixed solution of NBT (Roche) and BCIP (Roche), according to the manufacturer's instructions.

Cell isolation and culture. PBMCs were separated from the peripheral blood of healthy controls and psoriasis patients by density gradient centrifugation (GE Healthcare). $\mathrm{CD} 4^{+} \mathrm{T}$ cells were isolated by positive selection using Miltenyi beads according to the manufacturer's instructions (Miltenyi Biotec) in sterile or nonsterile conditions. Then, the cells were cultured in RPMI 1640 medium (Gibco) with $10 \%$ fetal bovine serum (FBS, HyClone) at $37^{\circ} \mathrm{C}$ and $5 \% \mathrm{CO}_{2}$ or collected directly for subsequent experiments. HEK293T cells were maintained in DMEM (Gibco) supplemented with 10\% FBS (HyClone).

siRNAs, plasmids, and agomir/antagomir transfection of $T$ cells. For siRNAs and plasmid transfections, $\mathrm{CD}^{+} \mathrm{T}$ cells were transfected with siRNA or plasmid using the Human T Cell Nucleofector Kit and Amaxa Nucleofector system (Lonza). Briefly, total CD4 $4^{+} \mathrm{T}$ cells were harvested and resuspended in $100 \mu \mathrm{l}$ human T cell nucleofector solution, and $10 \mu \mathrm{l} 20 \mu \mathrm{M}$ siRNA or $5 \mu \mathrm{g}$ plasmid was added. Cells were electrotransfected using the nucleofector program V-024 in the Amaxa Nucleofector apparatus (Lonza) and cultured in RPMI 1640 complete medium (Gibco). Cell culture media were refreshed 6 hours after transfection. For agomir/antagomir transfections, total $\mathrm{CD} 4^{+} \mathrm{T}$ cells or naive $\mathrm{CD}^{+} \mathrm{T}$ cells were cultured in $1 \mathrm{ml}$ Opti-MEM (Gibco) with $2 \mu$ l of $200 \mu \mathrm{M}$ agomir-210/antagomir-210 or their corresponding controls. After 6 hours, $1 \mathrm{ml}$ RPMI 1640 complete medium (Gibco) was added to the wells and cells were cultured for different time points (the final concentration of agomir/antagomir was $200 \mathrm{nM}$ ). The cells were harvested for subsequent experiments. The STAT6, LYN, and HIF-1a expression plasmids and the empty control plasmid were purchased from Vigene Biosciences Inc. P300, HIF-1 $\alpha$, STAT6, and LYN siRNAs and negative control siRNAs were purchased from Thermo Fisher Scientific. Related siRNA sequences are listed in Supplemental Table 2.

Cytokine stimulation of $\mathrm{CD} 4^{+} \mathrm{T}$ cells. Normal human $\mathrm{CD} 4^{+} \mathrm{T}$ cells were stimulated with TGF- $\beta$ (5 ng/ml, PeproTech), IL-23 (20 ng/ml, PeproTech), IL-6 (10 ng/ml, PeproTech), or IL-1 1 (10 ng/ml, PeproTech). Cells were collected at 0, 6, 12, 24, and 48 hours to perform RTqPCR or Western blot.

In vitro human $T$ cell differentiation. PBMCs were separated from the peripheral blood of healthy controls by density gradient centrifugation (GE Healthcare). $\mathrm{CD} 4^{+} \mathrm{CDRA}^{+} / \mathrm{RO}^{-}$naive $\mathrm{T}$ cells were isolated by negative selection using Miltenyi beads according to the manufacturer's instructions. The purity of the enriched subset was validated by flow cytometry and was generally higher than $95 \%$. Purified naive T cells were stimulated with plate-bound anti-CD3 $(5 \mu \mathrm{g} / \mathrm{ml}$, Calbiochem, catalog 217570) and anti-CD28 (2 $\mu \mathrm{g} / \mathrm{ml}$, Calbiochem, catalog 217669) for 5 days under the following polarizing conditions: antiIFN- $\gamma(10 \mu \mathrm{g} / \mathrm{ml}$, eBioscience, catalog 16-7317-85) and anti-IL-4 (10 $\mu \mathrm{g} / \mathrm{ml}$, eBioscience, catalog 16-7048-85) for Th0; IL-12 (10 ng/ml,
PeproTech) and anti-IL-4 (10 $\mu \mathrm{g} / \mathrm{ml})$ for Th1; IL-4 (2.5 ng/ml, PeproTech) and anti-IFN- $\gamma(10 \mu \mathrm{g} / \mathrm{ml})$ for Th2; TGF- $\beta$ ( $5 \mathrm{ng} / \mathrm{ml}$, PeproTech), IL-6 (10 ng/ml, PeproTech), IL-1 $\beta$ (10 ng/ml, PeproTech), IL-23 (20 $\mathrm{ng} / \mathrm{ml}$, PeproTech), anti-IFN- $\gamma(10 \mu \mathrm{g} / \mathrm{ml})$ and anti-IL-4 $(10 \mu \mathrm{g} / \mathrm{ml})$ for Th17; TGF- $\beta$ (5 ng/ml) and IL-2 (10 ng/ml, PeproTech) for iTreg. We performed cell culture in 24-well plates with a total volume of $1 \mathrm{ml} /$ well of culture medium with $1 \times 10^{6}$ naive $\mathrm{CD} 4^{+} \mathrm{T}$ cells. The medium was refreshed on day 3 .

In vitro mouse $T$ cell differentiation. Naive $\mathrm{CD} 4^{+} \mathrm{T}$ cells were purified from pooled single-cell suspensions of spleen using a mouse naive $\mathrm{CD}^{+} \mathrm{T}$ cell isolation kit II (Miltenyi Biotec); the purity of the enriched subset was validated by flow cytometry and was generally higher than 95\%. Purified naive CD4 ${ }^{+} \mathrm{T}$ cells were stimulated with plate-bound anti-CD3 (5 $\mu \mathrm{g} / \mathrm{ml}$, eBioscience, catalog 16-0031-85) and anti-CD28 ( $2 \mu \mathrm{g} / \mathrm{ml}$, eBioscience, catalog 16-0281-85) for 5 days under different polarizing conditions. For Th0 polarization, naive $\mathrm{CD}^{+} \mathrm{T}$ cells were activated with anti-IFN- $\gamma(10 \mu \mathrm{g} / \mathrm{ml}$, eBioscience, catalog 16-731285) and anti-IL-4 (10 $\mu \mathrm{g} / \mathrm{ml}$, eBioscience, catalog 16-7041-85). For Th1 polarization, naive CD4 ${ }^{+} \mathrm{T}$ cells were activated with IL-12 (10 $\mathrm{ng} / \mathrm{ml}$, PeproTech) in the presence of anti-IL-4 (10 $\mu \mathrm{g} / \mathrm{ml})$. For Th2 polarization, naive $\mathrm{CD}^{+}{ }^{+} \mathrm{T}$ cells were activated with $\mathrm{IL}-4(20 \mathrm{ng} / \mathrm{ml}$, PeproTech) in the presence of anti-IFN- $\gamma(20 \mu \mathrm{g} / \mathrm{ml})$. For Th17 cell differentiation, naive CD $4^{+}$T cells were stimulated with TGF- $\beta$ ( $2 \mathrm{ng} /$ ml, PeproTech), IL-6 (30 ng/ml, PeproTech), IL-1 $\beta$ (10 ng/ml, PeproTech), and IL-23 (20 ng/ml, R\&D Systems) in the presence of antiIFN- $\gamma(10 \mu \mathrm{g} / \mathrm{ml})$ and anti-IL-4 $(10 \mu \mathrm{g} / \mathrm{ml})$. For iTreg differentiation, naive $\mathrm{CD}^{+} \mathrm{T}$ cells were activated with TGF- $\beta$ ( $\left.5 \mathrm{ng} / \mathrm{ml}\right)$ and IL-2 (10 $\mathrm{ng} / \mathrm{ml}$, PeproTech) in the presence of anti-IFN- $\gamma(5 \mu \mathrm{g} / \mathrm{ml})$ and antiIL-4 $(5 \mu \mathrm{g} / \mathrm{ml})$. We performed cell culture in 24 -well plates with a total volume of $1 \mathrm{ml} /$ well of culture medium with $1 \times 10^{6}$ naive $\mathrm{CD} 4^{+} \mathrm{T}$ cells. The medium was refreshed on day 3.

Flow cytometry. Cytokines, transcription factors, and surface markers were evaluated using a FACSCanto II (BD Biosciences). In brief, for cytokine detection, cells were stimulated for 4 hours with PMA and ionomycin with the addition of GolgiPlug (BD Biosciences). Then, cells were incubated with antibodies against surface markers on ice for 30 minutes in the dark. For intracellular staining, cells were fixed and permeabilized with a Human Foxp3 Buffer Set (BD Biosciences) or Cytofix/Cytoperm (BD Biosciences) and stained with fluorescent antibodies for an additional 30 minutes on ice in the dark. Events were collected and analyzed with FlowJo software (Tree Star). The following antibodies were used: FITC anti-human CD4 (BioLegend, catalog 317408), PE anti-human IL-17A (BD Biosciences, cata$\log 560487$ ), Alexa Fluor 647 anti-human IFN- $\gamma$ (BioLegend, catalog 502516), PE anti-human IL-4 (BioLegend, catalog 500704), Alexa Fluor 647 anti-human FOXP3 (BioLegend, catalog 320114), PE antihuman CD25 (eBioscience, catalog 12-0259-42), FITC anti-mouse CD4 (BioLegend, catalog 100406), APC-Cy7 anti-mouse CD4 (BioLegend, catalog 100414), PerCP-Cy5.5 anti-mouse IFN- $\gamma$ (BD Biosciences, catalog 560660), APC anti-mouse IL-4 (BD Biosciences, catalog 562045), PE anti-mouse IL-4 (BioLegend, catalog 504104), PE anti-mouse IL-17A (BioLegend, catalog 561020), Alexa Fluor 647 anti-mouse IL-17A (BioLegend, catalog 506912), PE anti-mouse CD25 (BioLegend, catalog 102008), and Alexa Fluor 647 anti-mouse FOXP3 (BioLegend, catalog 126408).

Confocal assay. $\mathrm{CD}^{+} \mathrm{T}$ cells were fixed and permeabilized with a Human Foxp3 Buffer Set or Cytofix/Cytoperm Plus Kit based on the 
manufacturer's instructions and intracellularly stained with rabbit anti-STAT6 (Abcam, catalog ab32520) or mouse anti-LYN (Abcam, catalog ab1890) at $4^{\circ} \mathrm{C}$ in the dark for 1 hour. Then the cells were incubated with Alexa Fluor 594-conjugated donkey anti-rabbit IgG $(\mathrm{H}+\mathrm{L})$ secondary Ab (Life Technologies, catalog R37119) or Alexa Fluor 488conjugated goat anti-mouse IgG $(\mathrm{H}+\mathrm{L})$ secondary Ab (Life Technologies, catalog R37120) at $4^{\circ} \mathrm{C}$ for 30 minutes in the dark. Then, the cells were analyzed using a Zeiss LSM 780.

$R T-q P C R$. Total RNA was extracted from cells or skin samples using TRIzol reagent (Invitrogen) and a NanoDrop spectrophotometer (ND-2000, Thermo Fisher Scientific) was used for RNA quality control. For epidermal RNA extraction, an overnight incubation of skin samples at $4^{\circ} \mathrm{C}$ in dispase II $(2.5 \mathrm{U} / \mathrm{ml})$ (Roche) was performed to separate the epidermis from the dermis. mRNA was reverse-transcribed with the PrimeScript RT reagent Kit with gDNA Eraser (TaKaRa Biotech Co.) and each test consumed $1 \mu \mathrm{g}$ of total RNA according to the manufacturer's instructions. miR-210 was specifically reversetranscribed with the Ribobio Bulge-Loop miRNA RT-qPCR kit, along with U6. qPCR was carried out with the SYBR Premix Ex Taq II (Tli RNaseH Plus) (TaKaRa Biotech Co.) using a LightCycler 96 (Roche) thermocycler. The relative expression level of miR or gene was calculated by $2^{\text {-(CtmiR-210/gene-CtU6/GAPDH) }}$, which normalized to the internal controls U6 (miR) or GAPDH (gene). The fold change of gene or miR expression was calculated by $2^{-(\Delta C \text { Ctexperimental group- } \Delta \text { Ctcontrol group) }}$, which normalized to the control group. Primers for miR-210 and U6 were purchased from RiboBio Co., and other relevant primers are shown in Supplemental Table 2.

Western blotting. Cells or skin samples were lysed in radio immunoprecipitation assay (RIPA) buffer supplemented with protease and phosphatase inhibitor (Beyotime). Proteins were quantified by the Bradford assay (HyClone-Pierce). Rabbit anti-HIF-1 $\alpha$ Ab (1:1,000; Cell Signal Technology, catalog 14179), rabbit anti-STAT6 Ab (1:1,000; Cell Signal Technology, catalog 9362), mouse anti-LYN Ab (1:1,000; Abcam, catalog ab1890), mouse anti- $\beta$-actin Ab (1:2,000, Abcam, catalog ab6276) or goat anti-GAPDH Ab (1:2,000, Abcam, catalog ab9483) was used. Data were analyzed using a GE-ImageQuant LAS 4000 mini (GE Healthcare). Quantification of HIF-1 $\alpha$, STAT6, and LYN was normalized to GAPDH by densitometry. Images have been cropped for presentation.

ChIP-qPCR. ChIP assays were performed with a ChIP kit (Millipore). According to the protocol, $\mathrm{CD} 4^{+} \mathrm{T}$ cells were fixed in $1 \%$ formaldehyde and then lysed for 10 minutes on ice. Chromatin was sheared by sonication. After the cell lysates were precleared with protein $G$ agarose beads, they were immunoprecipitated overnight at $4^{\circ} \mathrm{C}$ with anti-HIF-1 $\alpha$ (Cell Signaling Technology, catalog 14179), anti-P300 (Abcam, catalog ab10485), and anti-acetyl-histone H3 (Active Motif, catalog 39139). Then, protein $G$ agarose beads were added, and the resulting mixture was rotated for 1 hour at $4^{\circ} \mathrm{C}$. After washing and elution, cross-links were reversed by heating at $65^{\circ} \mathrm{C}$ for 12 to 16 hours. The eluted DNA was purified, and the samples were analyzed by qPCR with input DNA (total chromatin) as an endogenous control. ChIPqPCR primer sequences are listed in Supplemental Table 2.

Th1/Th2/Th17 cytokines multi-analyte flow assay. Human psoriatic $\mathrm{CD}^{+} \mathrm{T}$ cells and normal human $\mathrm{CD} 4^{+} \mathrm{T}$ cells were transfected with antagomir-210 or agomir-210 followed by stimulation using platebound anti-CD3 (5 $\mu \mathrm{g} / \mathrm{ml}$, Calbiochem, catalog 217570) plus soluble anti-CD28 (2 $\mu \mathrm{g} / \mathrm{ml}$, Calbiochem, catalog 217669) and cultured in complete RPMI 1640 medium (Gibco). Supernatants were collected after 48 hours and subjected to cytokine assay. The concentrations of IL-17A, IL-17F, IFN- $\gamma$, and IL-4 were analyzed using a LEGENDplex Multi-Analyte Flow Assay Kit (BioLegend) according to the manufacturer's instructions.

Luciferase assay. For 3'-UTR reporter assays, the 3'-UTRs of the STAT6 and LYN mRNA containing WT or MU miR-210 binding sites were subcloned into the psiCHECK-2 vector (Promega). Then, $1 \times$ $10^{5}$ HEK293T cells were seeded in the wells of a 24-well plate 1 day before transfection, and each well was transfected with a mixture of $10 \mathrm{ng}$ 3'-UTR luciferase reporter vector (WT or MU) and agomir-210 or agomir control using Lipofectamine 2000 (Invitrogen) according to the manufacturer's instructions. Twenty-four hours after transfection, cells were lysed, and luciferase activity was measured on a luminometer (Promega) by using the Dual-Luciferase Reporter Assay System (Promega). The ratio of Renilla luciferase activity to firefly luciferase activity was calculated for each well.

For promoter reporter assay, 3 WT fragments, including S1 (from $-1,000$ bp to -501 bp, pGL3-S1), S2 (from -500 bp to -1 bp, pGL3-S2 or HIF- $1 \alpha-W T$ ) and S3 (from $-1,000$ bp to $-1 \mathrm{bp}, \mathrm{pGL3}-\mathrm{S} 3$ ), upstream of the TSS of miR-210 and fragment S2 with a mutant HIF- $1 \alpha$ binding sequence (HIF-1 $\alpha$-MU) were subcloned into the pGL3-basic vector (Promega). Then, $1 \times 10^{5}$ HEK293T cells were seeded in the wells of a 24-well plate 1 day before transfection, and each well was transfected with a mixture of $500 \mathrm{ng}$ pGL3 luciferase vector and $100 \mathrm{ng}$ pRL-TK Renilla vector with or without $1 \mu \mathrm{g}$ HIF-1 $\alpha$ expression plasmid or empty control plasmid. Twenty-four hours after transfection, cells were lysed, and luciferase activity was measured on a luminometer (Promega) using the Dual-Luciferase Reporter Assay System. The ratio of firefly luciferase to Renilla luciferase was calculated for each well.

Co-IP. Co-IP assays were performed with the Dynabeads Protein $\mathrm{G}$ Immunoprecipitation Kit (Invitrogen) based on the manufacturer's instructions. First, we collected the treated fresh cells and extracted protein using RIPA lysis buffer supplemented with protease and phosphatase inhibitors. A capture Ab that specifically recognizes the bait protein was added to the lysates, forming a new Ab-bait-target complex. The Ab was then used as a handle to immobilize the proteins on beads (Life Technologies). Proteins not immobilized on beads were removed by a series of washes. Finally, the bait-protein complexes were eluted from the beads and dissociated by boiling in SDS. The presence or absence of the target protein, which is the endpoint of the assay, was evaluated by Western blot. Rabbit anti-HIF-1 $\alpha$ Ab (Abcam, catalog ab51608), mouse anti-P300 Ab (Abcam, catalog ab3164), and mouse anti-Flag Ab (Abcam, catalog ab45766) were used.

Histological analysis. The histological analysis was performed as previously described $(16,53)$. Human and mouse skin tissues were fixed in formalin and embedded in paraffin. Sections $(6 \mu \mathrm{m})$ were stained with hematoxylin and eosin (H\&E) and stored at room temperature. Acanthosis and the number of dermis-infiltrating cells were assessed as histological features. Briefly, for measuring acanthosis, the epidermal area was outlined, and its pixel size was measured using the lasso tool in Adobe Photoshop CS4. The relative area of the epidermis was calculated using the following formula: area $=$ pixels $/$ (horizontal resolution $\times$ vertical resolution). For counting dermis-infiltrating cells, 6 areas ( 0.444 square inch for one) in $3 \mathrm{sec}-$ tions of each sample were randomly selected, and the number of infiltrating cells was calculated. 
RNA-binding protein immunoprecipitation (RIP). Immunoprecipitation of RNA binding protein-RNA complexes was performed using the Magna RIP RNA-Binding Protein Immunoprecipitation Kit (Millipore, catalog 17-701) according to the manufacturer's instructions. In brief, $\mathrm{CD}^{+} \mathrm{T}$ cells were isolated from psoriasis patients and healthy controls, and then cells were lysed by RIP lysis buffer and stored at $-80^{\circ} \mathrm{C}$. Magnetic beads were incubated with $5 \mu \mathrm{g}$ anti-Ago2 (Abcam, catalog ab32381) with rotation for 30 minutes at room temperature. Anti-SNRNP70 (Millipore, catalog CS203216) and rabbit IgG (Millipore, catalog PP64B) served as positive and negative controls, respectively. After rapid thawing and centrifugation at 18,000 $\mathrm{g}$ for $10 \mathrm{~min}$ utes, the supernatant was incubated with a bead-Ab complex overnight at $4^{\circ} \mathrm{C}$. Immunoprecipitated RNA was analyzed by qPCR. The primer sequences for STAT6 and LYN are shown in Supplemental Table 2.

Statistics. Statistical analysis was performed using GraphPad Prism 6.0. Data are presented as the mean \pm SEM. We assessed data for normal distribution and similar variance between groups. Statistical significance $\left({ }^{*} P<0.05,{ }^{* *} P<0.01,{ }^{* * *} P<0.001\right)$ was assessed using 2-tailed unpaired Student's $t$ test for comparisons between 2 groups and 1-way analysis of variance (ANOVA) with relevant post hoc tests for multiple comparisons. When the data were not normally distributed or displayed unequal variances between 2 groups, we used the 2-tailed Mann-Whitney $U$ test for statistical analysis. Correlation analysis was performed using Pearson's $r$ test or Spearman's $r$ test (for abnormally distributed data). No statistical method was used to predetermine the sample size. Animals were allocated at random to treatment groups.

Study approval. All human studies were approved by the ethics committee of the Second Xiangya Hospital of Central South University. Written informed consent was obtained from all subjects. All animal care protocols and experiments were reviewed and approved by the Animal Care and Use Committees of the Laboratory Animal Research Center at the Second Xiangya Medical School of Central South University.

\section{Author contributions}

RW and JZ performed most of the experiments, analyzed the data and wrote the manuscript. JY performed the in vitro human $\mathrm{T}$ cell differentiation. XD assisted with luciferase assays. YH and LC assisted with molecular cloning and ChIP experiments. PZ, HF, $\mathrm{ZL}$, and YS collected the clinical samples. ZW and XG assisted with in vitro mouse $\mathrm{T}$ cell differentiation experiments. $\mathrm{H}$. Wu assisted with flow cytometry and provided suggestions for the project. $\mathrm{H}$. Wang provided technical support and suggestions for the project. QL, MZ, and YS conceived the project and supervised the study. $\mathrm{MZ}$ and QL designed the experiments, analyzed and interpreted data, and wrote the manuscript.

\section{Acknowledgments}

This work was supported by the National Science Fund for Excellent Young Scholars (81522038) and the Project of Innovationdriven Plan of Central South University (2016CX029) to M. Zhao, the National Natural Science Foundation of China (81371743 and 81573051) to Y. Su, the Key Program of National Natural Science Foundation of China (81430074) to Q. Lu, and the Fundamental Research Funds for the Central Universities of Central South University (2016zzts143) to R. Wu.

Address correspondence to Qianjin Lu, The Second Xiangya Hospital of Central South University, 139 Renmin Middle Road, Changsha, Hunan 410011, China. Phone: 86.731.85295860; Email: qianlu5860@csu.edu.cn. Or to: Ming Zhao, The Second Xiangya Hospital of Central South University, 139 Renmin Middle Road, Changsha, Hunan 410011, China. Phone: 86.731.85292184; Email: zhaoming307@csu.edu.cn. Or to: Yuwen Su, The Second Xiangya Hospital of Central South University, 139 Renmin Middle Road, Changsha, Hunan 410011, China. Phone: 86.731.85295660; Email: suyuwen1963@csu.edu.cn.
1. Nestle FO, Kaplan DH, Barker J. Psoriasis. N Engl JMed. 2009;361(5):496-509.

2. Mak RK, Hundhausen C, Nestle FO. Progress in understanding the immunopathogenesis of psoriasis. Actas Dermosifiliogr. 2009;100 Suppl 2:2-13.

3. Becher B, Pantelyushin S. Hiding under the skin: Interleukin-17-producing $\gamma \delta$ T cells go under the skin? Nat Med. 2012;18(12):1748-1750.

4. Gutcher I, Becher B. APC-derived cytokines and $\mathrm{T}$ cell polarization in autoimmune inflammation. JClin Invest. 2007;117(5):1119-1127.

5. Krueger JG. Hiding under the skin: A welcome surprise in psoriasis. Nat Med. 2012;18(12):1750-1751.

6. Duhen T, Geiger R, Jarrossay D, Lanzavecchia A, Sallusto F. Production of interleukin 22 but not interleukin 17 by a subset of human skin-homing memory T cells. Nat Immunol. 2009;10(8):857-863.

7. Kagami S, Rizzo HL, Lee JJ, Koguchi Y, Blauvelt A. Circulating Th17, Th22, and Th1 cells are increased in psoriasis. J Invest Dermatol. 2010;130(5):1373-1383.

8. Fitzgerald O, Winchester R. Editorial: emerging evidence for critical involvement of the interleukin-17 pathway in both psoriasis and psoriatic arthritis. Arthritis Rheumatol.
2014;66(5):1077-1080.

9. Harrington LE, et al. Interleukin 17-producing $\mathrm{CD}^{+}{ }^{+}$effector $\mathrm{T}$ cells develop via a lineage distinct from the T helper type 1 and 2 lineages. Nat Immunol. 2005;6(11):1123-1132.

10. Mease PJ, Goffe BS, Metz J, VanderStoep A, Finck $\mathrm{B}$, Burge DJ. Etanercept in the treatment of psoriatic arthritis and psoriasis: a randomised trial. Lancet. 2000;356(9227):385-390.

11. Bartlett BL, Tyring SK. Ustekinumab for chronic plaque psoriasis. Lancet. 2008;371(9625):1639-1640.

12. Speeckaert R, van Geel N, Lambert J, Claeys L, Delanghe JR, Speeckaert MM. Secukinumab: IL-17A inhibition to treat psoriatic arthritis. Drugs Today. 2016;52(11):607-616.

13. Bartel DP. MicroRNAs: target recognition and regulatory functions. Cell. 2009;136(2):215-233.

14. Kuchen S, et al. Regulation of microRNA expression and abundance during lymphopoiesis. Immunity. 2010;32(6):828-839.

15. Xiao C, Rajewsky K. MicroRNA control in the immune system: basic principles. Cell. 2009;136(1):26-36.

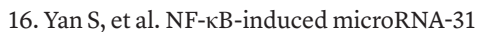
promotes epidermal hyperplasia by repressing protein phosphatase 6 in psoriasis. Nat Commun.
2015;6:7652.

17. Shen H, et al. MiR-330 inhibits IL-22-induced keratinocyte proliferation through targeting CTNNB1. Biomed Pharmacother. 2017;91:803-811.

18. Primo MN, Bak RO, Schibler B, Mikkelsen JG. Regulation of pro-inflammatory cytokines TNF $\alpha$ and IL24 by microRNA-203 in primary keratinocytes. Cytokine. 2012;60(3):741-748.

19. Fu D, et al. MicroRNA-138 regulates the balance of Th1/Th2 via targeting RUNX3 in psoriasis. Immunol Lett. 2015;166(1):55-62.

20. Zhao M, et al. Up-regulation of microRNA-210 induces immune dysfunction via targeting FOXP3 in CD4(+) T cells of psoriasis vulgaris. Clin Immunol. 2014;150(1):22-30.

21. van der Fits L, et al. Imiquimod-induced psoriasis-like skin inflammation in mice is mediated via the IL-23/IL-17 axis. J Immunol. 2009;182(9):5836-5845.

22. Hedrick MN, et al. CCR6 is required for IL-23induced psoriasis-like inflammation in mice. J Clin Invest. 2009;119(8):2317-2329.

23. Diani M, Altomare G, Reali E. T helper cell subsets in clinical manifestations of psoriasis. J Immunol Res. 2016;2016:7692024.

24. Lanford RE, et al. Therapeutic silencing of microRNA-122 in primates with chronic hepatitis $\mathrm{C}$ 


\section{RESEARCH ARTICLE}

virus infection. Science. 2010;327(5962):198-201.

25. Hou J, et al. Identification of miRNomes in human liver and hepatocellular carcinoma reveals miR-199a/b-3p as therapeutic target for hepatocellular carcinoma. Cancer Cell. 2011;19(2):232-243.

26. Krutzfeldt J, et al. Silencing of microRNAs in vivo with 'antagomirs'. Nature. 2005;438(7068):685-689.

27. Kim VN, Han J, Siomi MC. Biogenesis of small RNAs in animals. Nat Rev Mol Cell Biol. 2009;10(2):126-139.

28. Ajdukovic J. HIF-1 - a big chapter in the cancer tale. Exp Oncol. 2016;38(1):9-12.

29. Bedoya SK, Wilson TD, Collins EL, Lau K, Larkin $\mathrm{J} 3$ rd. Isolation and th17 differentiation of naive CD4 T lymphocytes. J Vis Exp. 2013;(79):e50765.

30. Agirre X, Martínez-Climent JÁ, Odero $\mathrm{MD}$, Prósper F. Epigenetic regulation of miRNA genes in acute leukemia. Leukemia. 2012;26(3):395-403.

31. Formosa A, et al. DNA methylation silences miR-132 in prostate cancer. Oncogene. 2013;32(1):127-134.

32. Zhang E, et al. H3K27 acetylation activatedlong non-coding RNA CCAT1 affects cell proliferation and migration by regulating SPRY4 and HOXB13 expression in esophageal squamous cell carcinoma. Nucleic Acids Res. 2017;45(6):3086-3101.

33. Cai H, et al. Epigenetic inhibition of miR- $663 \mathrm{~b}$ by long non-coding RNA HOTAIR promotes pancreatic cancer cell proliferation via up-regulation of insulin-like growth factor 2. Oncotarget. 2016;7(52):86857-86870.

34. Lai FF, Niu F, Yang HZ, Zhou WQ, Chen XG. [Development of a novel screening assay for inhibitors targeting HIF-1 $\alpha$ and P3OO interaction]. Yao Xue Xuе Bao. 2014;49(6):849-853.
35. Kyle HF, et al. Exploration of the HIF-1 $\alpha$ / p300 interface using peptide and Adhiron phage display technologies. Mol Biosyst. 2015;11(10):2738-2749.

36. Atilano ML, Glittenberg M, Monteiro A, Copley RR, Ligoxygakis P. MicroRNAs that contribute to coordinating the immune response inDrosophila melanogaster. Genetics. 2017;207(1):163-178.

37. Chu Q, Gao Y, Bi D, Xu T. MicroRNA-148 as a negative regulator of the common TLR adaptor mediates inflammatory response in teleost fish. Sci Rep. 2017;7(1):4124.

38. Zhu S, et al. The microRNA miR-23b suppresses IL-17-associated autoimmune inflammation by targeting TAB2, TAB3 and IKK- $\alpha$. Nat Med. 2012;18(7):1077-1086.

39. Luan L, Ding Y, Han S, Zhang Z, Liu X. An increased proportion of circulating Th22 and Tc22 cells in psoriasis. Cell Immunol. 2014;290(2):196-200.

40. Wang H, Flach H, Onizawa M, Wei L, McManus MT, Weiss A. Negative regulation of Hifla expression and TH17 differentiation by the hypoxia-regulated microRNA miR-210. Nat Immunol. 2014;15(4):393-401.

41. Rosenberger C, et al. Upregulation of hypoxiainducible factors in normal and psoriatic skin. J Invest Dermatol. 2007;127(10):2445-2452.

42. Lau M, Tsantikos E, Maxwell MJ, Tarlinton DM, Anderson GP, Hibbs ML. Loss of STAT6 promotes autoimmune disease and atopy on a susceptible genetic background. J Autoimmun. 2012;39(4):388-397.

43. Summers SA, Phoon RK, Odobasic D, Dewage L, Kitching AR, Holdsworth SR. Signal transducer and activation of transcription 6 (STAT6) regulates Thelper type 1 (Th1) and Th17 nephritogenic immunity in experimental cres-
The Journal of Clinical Investigation

centic glomerulonephritis. Clin Exp Immunol. 2011;166(2):227-234.

44. Hibbs ML, et al. Multiple defects in the immune system of Lyn-deficient mice, culminating in autoimmune disease. Cell.1995;83(2):301-311.

45. Tsantikos E, Oracki SA, Quilici C, Anderson GP, Tarlinton DM, Hibbs ML. Autoimmune disease in Lyn-deficient mice is dependent on an inflammatory environment established by IL-6. J Immunol. 2010;184(3):1348-1360.

46. Janas ML, Hodgkin P, Hibbs M, Tarlinton D. Genetic evidence for Lyn as a negative regulator of IL-4 signaling. J Immunol. 1999;163(8):4192-4198.

47. Odom S, et al. Negative regulation of immunoglobulin E-dependent allergic responses by Lyn kinase. J Exp Med. 2004;199(11):1491-1502.

48. Zhang P, Su Y, Zhao M, Huang W, Lu Q. Abnormal histone modifications in PBMCs from patients with psoriasis vulgaris. Eur J Dermatol. 2011;21(4):552-557.

49. Sun H, Kennedy PJ, Nestler EJ. Epigenetics of the depressed brain: role of histone acetylation and methylation. Neuropsychopharmacology. 2013;38(1):124-137.

50. Nograles KE, et al. Th17 cytokines interleukin (IL)-17 and IL-22 modulate distinct inflammatory and keratinocyte-response pathways. Br J Dermatol. 2008;159(5):1092-1102.

51. Büchau AS, Gallo RL. Innate immunity and antimicrobial defense systems in psoriasis. Clin Dermatol. 2007;25(6):616-624.

52. Obernosterer G, Martinez J, Alenius M. Locked nucleic acid-based in situ detection of microRNAs in mouse tissue sections. Nat Protoc. 2007;2(6):1508-1514.

53. Zhu H, et al. RIG-I antiviral signaling drives interleukin-23 production and psoriasis-like skin disease. EMBO Mol Med. 2017;9(5):589-604. 